\title{
New Classes of Partial Geometries and Their Associated LDPC Codes
}

\author{
Qiuju Diao, Member, IEEE, Juane Li, Student Member, IEEE, \\ Shu Lin, Life Fellow, IEEE, and Ian Blake, Life Fellow, IEEE
}

\begin{abstract}
The use of partial geometries to construct parity-check matrices for LDPC codes has resulted in the design of successful codes with a probability of error close to the Shannon capacity at bit error rates down to $10^{-15}$. Such considerations have motivated this further investigation. A new and simple construction of a type of partial geometries with quasi-cyclic structure is given and their properties are investigated. The trapping sets of the partial geometry codes were considered previously using the geometric aspects of the underlying structure to derive information on the size of allowable trapping sets. This topic is further considered here. Finally, there is a natural relationship between partial geometries and strongly regular graphs. The eigenvalues of the adjacency matrices of such graphs are well known and it is of interest to determine if any of the Tanner graphs derived from the partial geometries are good expanders for certain parameter sets, since it can be argued that codes with good geometric and expansion properties might perform well under message-passing decoding.
\end{abstract}

\section{Index Terms}

LDPC codes, partial geometries, strongly regular graphs, protographs, expander graphs

\section{INTRODUCTION}

Partial geometries play an important role in the construction of low-density parity-check (LDPC) codes [16], [32], [33] which currently give the most promising coding technique for

Q. Diao is with Sandisk Corp., Milpitas, CA 95035

Email:judiao@ucdavis.edu

J. Li and S. Lin are with ECE Dept., UC Davis, Davis, CA 95616

Email: \{jueli, shulin\}@ece.ucdavis.edu

Ian F. Blake is with ECE Dept. UBC, Vancouver, BC V6T 1 Z4

Email: ifblake@ece.ubc.ca

This work was supported by the NSF under Grant CCF-1015548. 
error control in communication and data storage systems due to their capacity-approaching performances and practically implementable decoding algorithms. Partial geometries are members of a broad class of combinatorial configurations with geometric properties referred to as finite geometries. The first classes of LDPC codes based on partial geometries were constructed based on Euclidean and Projective geometries over finite fields [24]. These classes of finite geometry LDPC codes have an abundance of algebraic and geometric structures and perform well with iterative decoding algorithms [24], [32], [33]. The construction of LDPC codes given in [24] was later generalized in different directions [22], [42], [43], [53], which resulted in several large classes of finite geometry LDPC codes. Codes based on the more general partial geometries were presented in [20], [21], [28], [48]. In a recent paper [11], it was shown that diverse classes of LDPC codes that appear in the literature are actually partial geometry codes although their construction methods were not based on geometric notions.

In this paper, aspects of partial geometries and their use in coding theory are considered. A characterization of a special category of partial geometries realized from an array of cyclic permutation matrices is given. The graph representation of a partial geometry is also described. Two new classes of partial geometries are constructed, one from prime fields and the other one from cyclic subgroups of prime orders of finite fields. New classes of quasi-cyclic (QC) LDPC codes are constructed based on these new constructions of partial geometries. The problem of determining the sizes of trapping sets of such geometric codes, initiated in [11], is continued here for both the partial geometries and the subclass of generalized quadrangles (GQs). Finally, since the eigenvalues of the adjacency matrices of all the geometric objects under investigation are known, the expansion properties of them can be determined and are summarized to consider the possibility that they may play a role in suggesting promising candidates for LDPC codes for further investigation. Such candidates would have to be verified by simulation.

\section{Definitions, Concepts and Structural Properties of Partial Geometries}

Consider a system composed of a set $\mathbf{N}$ of $n$ points and a set $\mathbf{M}$ of $m$ lines where each line is a set of points. If a line $L$ contains a point $v$, we say that $v$ is on $L$ and that $L$ passes through $v$. If two points are on a line, then we say that the two points are adjacent and if two lines pass through the same point, then we say that the two lines intersect, otherwise they are parallel. The system composed of the sets $\mathbf{N}$ and $\mathbf{M}$ is a partial geometry [5], [6], [8] if the following conditions are satisfied for some fixed integers $\gamma \geq 2, \rho \geq 2$ and $\delta \geq 1$ : 
1) Any two points are on at most one line;

2) Each point is on $\gamma$ lines;

3) Each line passes through $\rho$ points;

4) If a point $v$ is not on a line $L$, then there are exactly $\delta$ lines, each passing through $v$ and a point on $L$.

Such a partial geometry will be denoted by $\operatorname{PaG}(\gamma, \rho, \delta)$ and $\gamma, \rho$ and $\delta$ are called the parameters of the partial geometry. The parameter $\delta$ is called the connection number of the geometry.

A simple counting argument [5] shows that the partial geometry $\operatorname{PaG}(\gamma, \rho, \delta)$ has exactly

$$
n=\rho((\rho-1)(\gamma-1)+\delta) / \delta
$$

points and

$$
m=\gamma((\rho-1)(\gamma-1)+\delta) / \delta
$$

lines.

If $v$ and $v^{\prime}$ are adjacent points, then there are exactly $\gamma \delta+\rho-\gamma-\delta-1$ points, such that each of these points is adjacent to both $v$ and $v^{\prime}$. On the other hand, if $v$ and $v^{\prime}$ are not adjacent, then there are exactly $\gamma \delta$ points, such that each of these points is adjacent to both $v$ and $v^{\prime}$. Each point $v$ is adjacent to $(\delta+1)(\rho-1)$ other points. Three adjacent non-colinear points form a triangle. It follows from the above adjacency property that two adjacent points $v$ and $v^{\prime}$ are on $\gamma \delta+\rho-\gamma-\delta-1$ triangles. As will be discussed later, such a triangle in the (point-point) adjacency matrix of the graph of the (point-point) partial geometry leads to a cycle of length 6 in the corresponding Tanner graph [44], assuming $\delta>1$.

Well known examples of partial geometries are Euclidean and projective geometries [9], [34] over finite fields. If $\delta=\gamma-1$, the partial geometry $\operatorname{PaG}(\gamma, \rho, \gamma-1)$ is called a net which consists of $n=\rho^{2}$ points and $m=\gamma \rho$ lines. Each point $v$ not on a line $L$ is on a unique line which is parallel to $L$. Equivalently, there is a unique line $L^{\prime}$ that intersects $v$ and $L$. The set of $m=\gamma \rho$ lines in the net $\operatorname{PaG}(\gamma, \rho, \gamma-1)$ can be partitioned into $\gamma$ classes, each consisting of $\rho$ lines, such that all the lines in each class are parallel, any two lines in two different classes intersect, and each of the $n=\rho^{2}$ points is on a unique line in each class. These classes of lines are called parallel bundles. A two-dimensional Euclidean geometry (or affine geometry) [34] is a net.

For every point $v$ in $\operatorname{PaG}(\gamma, \rho, \gamma-1)$, there are exactly $\gamma$ lines that intersect at $v$, i.e., lines that pass through $v$. These lines are said to form an intersecting bundle at $v$, denoted by $\Delta(v)$. 
Notice that $v$ is on every line in $\Delta(v)$, there are exactly $\gamma(\rho-1)$ points, each is on a unique line in $\Delta(v)$, and all the other $n-\gamma(\rho-1)-1$ points in $\operatorname{PaG}(\gamma, \rho, \gamma-1)$ are not on any line in $\Delta(v)$. If $\delta=\rho$, then every point in $\operatorname{PaG}(\gamma, \rho, \rho)$ is adjacent to $v$ since every point is on a line in $\Delta(v)$. In this case, any two points in $\operatorname{PaG}(\gamma, \rho, \rho)$ are connected by a line. Examples for which $\delta=\rho$ include two-dimensional Euclidean and projective geometries (also called affine and projective planes) [34].

Denote the points and lines in $\operatorname{PaG}(\gamma, \rho, \delta)$ by $v_{0}, v_{1}, \ldots, v_{n-1}$ and $L_{0}, L_{1}, \ldots, L_{m-1}$, respectively. Then, $\mathbf{N}=\left\{v_{0}, v_{1}, \ldots, v_{n-1}\right\}$ and $\mathbf{M}=\left\{L_{0}, L_{1}, \ldots, L_{m-1}\right\}$. Algebraically, a partial geometry $\operatorname{PaG}(\gamma, \rho, \delta)$ with $n$ points and $m$ lines is commonly represented by a $m \times n$ matrix $\mathbf{H}_{\mathrm{PaG}}=\left[h_{i, j}\right]_{0 \leq i<m, 0 \leq j<n}$ with 0 and 1 entries whose rows and columns correspond to the lines and points of $\operatorname{PaG}(\gamma, \rho, \delta)$, respectively. The rows are labeled from 0 to $m-1$ (or by the lines $\left\{L_{0}, L_{1}, \ldots, L_{m-1}\right\}$ in this order) and the columns are labeled from 0 to $n-1$ (or the points $\left\{v_{0}, v_{1}, \ldots, v_{n-1}\right\}$ in this order). The entry $h_{i, j}$ is 1 (i.e., $h_{i, j}=1$ ) if and only if the point $v_{j}$ labeled by $j$ (called the $j$-th point) is on the line $L_{i}$ labeled by $i$ (called the $i$-th line); otherwise, $h_{i, j}=0$. This matrix $\mathbf{H}_{\mathrm{PaG}}$ is called the line-point adjacency matrix of the partial geometry $\operatorname{PaG}(\gamma, \rho, \delta)$ which shows the incidence relationship of the lines and points of $\operatorname{PaG}(\gamma, \rho, \delta)$. The $i$-th row of $\mathbf{H}_{\mathrm{PaG}}$ is called the incidence vector of the $i$-th line $L_{i}$. The transpose $\mathbf{H}_{\mathrm{PaG}}^{T}$ of $\mathbf{H}_{\mathrm{PaG}}$ is also an adjacency matrix of $\operatorname{PaG}(\gamma, \rho, \delta)$ called the point-line adjacency matrix of $\operatorname{PaG}(\gamma, \rho, \delta)$.

Graphically, a partial geometry $\operatorname{PaG}(\gamma, \rho, \delta)$ can be displayed by an adjacency graph $\mathcal{G}(\gamma, \rho, \delta)$ with its $n$ points represented by $n$ nodes or vertices, labeled by $v_{0}, v_{1}, \ldots, v_{n-1}$. Two vertices $v_{j}$ and $v_{k}$ are connected by an edge $\left(v_{j}, v_{k}\right)$ if and only if they are on the same line. A line $L=\left\{v_{j_{0}}, v_{j_{1}}, \ldots, v_{j_{\rho-1}}\right\}$ in $\operatorname{PaG}(\gamma, \rho, \delta)$ is represented by a sequence of $\rho-1$ connected edges, $\left(v_{j_{0}}, v_{j_{1}}\right),\left(v_{j_{1}}, v_{j_{2}}\right), \ldots,\left(v_{j_{\rho-2}}, v_{j_{\rho-1}}\right)$. Each node $v_{j_{k}}$ in $\mathcal{G}(\gamma, \rho, \delta)$ has $\gamma$ edges incident with it and $\gamma$ is called the degree of $v_{j_{k}}$. It is the number of lines in $\operatorname{PaG}(\gamma, \rho, \delta)$ that intersect at the point $v_{j_{k}}$.

The partial geometry $\operatorname{PaG}(\gamma, \rho, \delta)$ can also be represented by a bipartite graph [41], denoted by $\mathcal{G}(\mathbf{V}, \mathbf{C})$, which is more commonly used in coding theory. It consists of two sets of nodes, denoted by $\mathbf{V}$ ( the set of variable nodes (VNs)) and $\mathbf{C}$ (the set of check nodes (CNs)), of size $n$ and $m$, respectively. The $n$ nodes in $\mathbf{V}$, denoted by $v_{0}, v_{1}, \ldots, v_{n-1}$, represent the $n$ points in $\operatorname{PaG}(\gamma, \rho, \delta)$ with $v_{j}$ representing the point $v_{j}$ and the $m$ nodes in $\mathbf{C}$, denoted by $c_{0}, c_{1}, \ldots, c_{m-1}$, represent the $m$ lines in $\operatorname{PaG}(\gamma, \rho, \delta)$ with $c_{i}$ representing the line $L_{i}$. A node $v_{j}$ in $\mathbf{V}$ is connected to a node $c_{i}$ in $\mathbf{C}$ if and only if the point $v_{j}$ in $\operatorname{PaG}(\gamma, \rho, \delta)$ represented by the node $v_{j}$ is on 
the line $L_{i}$ in $\operatorname{PaG}(\gamma, \rho, \delta)$ represented by the node $c_{i}$. This bipartite graph $\mathcal{G}(\mathbf{V}, \mathbf{C})$ is called the Tanner graph [44] associated with the line-point adjacency matrix $\mathbf{H}_{\mathrm{PaG}}$ of the partial geometry $\operatorname{PaG}(\gamma, \rho, \delta)$, commonly used in the study of LDPC codes in coding theory [44]. We call this bipartite graph a $(\gamma, \rho)$ - biregular bipartite graph since the VNs on the left are regular (have the same degree $\gamma$ ) and the CNs on the right are regular (of the same degree $\rho$ ). The associated (or the adjacency) matrix $\mathbf{H}_{\mathrm{PaG}}$ is used as the parity-check matrix of the code.

The girth of the $(\gamma, \rho)$-biregular bipartite graph $\mathcal{G}(\mathbf{V}, \mathbf{C})$ (defined as length of the shortest cycle) of the partial geometry $\operatorname{PaG}(\gamma, \rho, \delta)$ for $\delta>1$, is 6 and there are $m \gamma(\gamma-1)(\delta-1)(\rho-1) / 6$ cycles of length 6 in $\mathcal{G}(\mathbf{V}, \mathbf{C})$ [21]. For $\delta=1$ the partial geometry is a generalized quadrangle (GQ) [35], and the corresponding graph will have girth of 8. A simple modification of the argument of [21] to find the number of cycles of length 8 is as follows. Consider a line $L$ and points $v_{1}, v_{2}$ on $L$. Consider the intersecting bundles of lines, $\Delta\left(v_{1}\right)$ and $\Delta\left(v_{2}\right)$. Each set contains $(\gamma-1)$ lines other than $L$. Consider a point $v$ on a line $L_{2}$ through $v_{2}$ other than $L$ (i.e., in $\left.\Delta\left(v_{2}\right) \backslash\{L\}\right)$ and a line $L_{1}$ through $v_{1}$ in $\Delta\left(v_{1}\right) \backslash\{L\}$. By the definition of a GQ, there is a unique line through $v$ that intersects $L_{1}$. This is true for each point on the lines in $\Delta\left(v_{2}\right)$, other than $v_{2}$ and each line in $\Delta\left(v_{1}\right)$ other than $L$. Hence, there are $(\gamma-1)^{2}(\rho-1)$ distinct quadrangles in the GQ that contain $v_{1}, v_{2}$ as a side and $\left(\begin{array}{c}\rho \\ 2\end{array}\right)$ ways of choosing the two points on a line. The argument is repeated for each of the $m$ lines in the geometry and note that each quadrangle is repeated four times in this count giving the number of quadrangles in the geometry (cycles of length 8 in the Tanner graph) as

$$
m(\rho-1)(\gamma-1)^{2}\left(\begin{array}{l}
\rho \\
2
\end{array}\right) / 4 .
$$

\section{A Special Category of Partial Geometries}

In this section, we present a special category of partial geometries which are specified by their line-point adjacency matrices. First, we prove the conditions for a matrix of a specific type to be the line-point adjacency matrix of a partial geometry. Some structural properties of this category of partial geometries are then developed. In Sections $\mathrm{V}$ and VI, we will present two methods for constructing this category of partial geometries based on prime fields and cyclic subgroups of prime orders of finite fields, respectively. 


\section{A. Line-Point Adjacency Matrices}

Consider an $m \times n$ matrix $\mathbf{H}$ with 0- and 1-entries for which two rows (or two columns) have at most one place where both have 1 entries. Such a constraint on rows and columns are called the row and column constraint (RC-constraint) [24]. The matrix $\mathbf{H}$ is called an $R C$-constrained matrix. An RC-constrained matrix $\mathbf{H}$ is $(\gamma, \rho)$-biregular if each of its $n$ columns has $\gamma$ 1-entries and each of its $m$ rows has $\rho$ 1-entries. The parameters $\gamma$ and $\rho$ are called the column and row weights of $\mathbf{H}$, respectively. Suppose the rows of a $(\gamma, \rho)$-biregular matrix $\mathbf{H}$ are labeled from 0 to $m-1$ and the columns from 0 to $n-1$. The $\gamma$ rows of $\mathbf{H}$ that have 1-entries at the $j$-th position with $0 \leq j<n$ are said to be attached to the $j$-th column. Likewise, the $\rho$ columns that have 1 -entries in the $i$-th row are said to be attached to the $i$-th row.

The following theorem gives the conditions that an RC-constrained $m \times n$ regular matrix $\mathbf{H}$ with 0 - and 1-entries is the line-point adjacency matrix of a partial geometry with $n$ points and $m$ lines.

Theorem 1. Let $\mathbf{H}$ be an $m \times n R C$-constrained $(\gamma, \rho)$-biregular matrix which is a $\gamma \times \rho$ array of $\gamma \times \gamma$ circulant permutation matrices (CPMs), where $m=\gamma^{2}$ and $n=\gamma \rho$. Then, $\mathbf{H}$ is the linepoint adjacency matrix of a partial geometry $\operatorname{Pa} G(\gamma, \rho, \rho-1)$ that has $n$ points corresponding to the columns of $\mathbf{H}$ and $m$ lines corresponding to the rows of $\mathbf{H}$.

Proof: Since $\mathbf{H}$ is a $\gamma \times \rho$ array of $\gamma \times \gamma$ CPMs, it is a $\gamma^{2} \times \gamma \rho(\gamma, \rho)$-biregular matrix with constant column weight $\gamma$ and constant row weight $\rho$. It is clear that each point (corresponding to a column) is on $\gamma$ lines (corresponding to $\gamma$ rows) and each line (corresponding to a row) passes through $\rho$ points. The RC-constraint implies that any two points are on at most one line.

It remains to show that if a point $v$ is not on a line $L$, then there are exactly $\rho-1$ lines that pass through $v$ and $\rho-1$ points on $L$. We group the $n$ points into $\rho$ sets of size $\gamma$ where the points in each set correspond to $\gamma$ consecutive columns in $\mathbf{H}$, namely, the columns comprising a column-block of CPMs in H. Since every row has $\rho$ ones, then by adding all the rows attached to the column corresponding to the point $v$, where the sum is over the integers rather than over $\mathrm{GF}(2)$, we obtain a vector, $\mathbf{z}$, of length $n$ whose components as integers add up to $\rho \gamma$. Notice that the entry in the column corresponding to the point $v$ in $\mathbf{z}$ is $\gamma$ while all other entries in columns corresponding to the $\gamma-1$ other points in the same set as $v$ are zeros since every row attached to the column corresponding to $v$ has zeros in all columns corresponding to other 
points in the same set as $v$ as every CPM has a single 1 in each row. Hence, all the $(\rho-1) \gamma$ components in $\mathbf{z}$ other than those corresponding to $v$ and the $\gamma-1$ points in the same set as $v$ add up to $(\rho-1) \gamma$. Because of the RC-constraint, all these components are at most equal to 1 implying that all of them are equal to 1 . Hence, every column other than those corresponding to $v$ or other points in its set is attached to a unique row corresponding to a line passing through $v$ while every column corresponding to a point other than $v$ but in the same set as $v$ is not attached to any row corresponding to a line passing through $v$. Since $L$ passes through a single point in each set, it passes through $\rho-1$ points in sets other than that of $v$. Each of these points is on a line passing through $v$. The point on $L$ that is in the same set as $v$ is not adjacent to $v$. Hence, there are exactly $\rho-1$ lines that pass through $v$ and $\rho-1$ points on $L$. This proves that $\mathbf{H}$ is the adjacency matrix of a partial geometry $\operatorname{PaG}(\gamma, \rho, \rho-1)$.

The proof of the above theorem gives insight into the structure of the partial geometry $\operatorname{PaG}(\gamma, \rho, \rho-1)$ which is discussed further in following sections.

It is of interest to develop characterizations of partial geometries in the sense that if a combinatorial construction is developed, what technique could be used to verify that it is a partial geometry? The next discussion considers one such approach for a particular case where the adjacency matrix of the configuration is an array of CPMs.

Let $\mathbf{H}$ be the $m \times n$ line-point adjacency matrix of a partial geometry $\operatorname{PaG}(\gamma, \rho, \delta)$. Suppose $m=m^{\prime} \ell$ and $n=n^{\prime} \ell$ and assume that $\mathbf{H}$ is an $m^{\prime} \times n^{\prime}$ array of CPMs of order $\ell$, i.e., if $\mathbf{P}$ is a generator of the cyclic matrix group with the first (or the top) row the $\ell$-tuple $(0,1,0, \cdots, 0)$. The group of such $\ell \times \ell$ matrices is $\left\{\mathbf{I}, \mathbf{P}, \ldots, \mathbf{P}^{\ell-1}\right\}, \mathbf{P}^{\ell}=\mathbf{I}$, where $\mathbf{I}$ is an $\ell \times \ell$ identity matrix.

The matrix $\mathbf{H H}^{T}$ is an $m \times m$ matrix of 0 's and 1 's whose $(i, j)$-th element is 1 if the $i$-th line intersects the $j$-th line and 0 if the lines do not intersect and with diagonal elements $\rho$. If the $i$-th column-block of CPMs of $\mathbf{H}$ is multiplied by the $\ell \times \ell$ matrix $\mathbf{I}+\mathbf{P}^{j}, 0 \leq i<n^{\prime}$ and $0 \leq j<\ell$, the effect is to add to the circulant in a given row of circulants, the power of a nonunity permutation (circulant), i.e., it adds a 1 to each row of the circulant in the $i$-th column-block of circulants, not in the position of the existing 1 . For $0 \leq i<n^{\prime}$ and $0 \leq j<\ell$, form the following $n^{\prime} \times n^{\prime}$ diagonal array of circulants of size $\ell \times \ell$ :

$$
\mathbf{D}^{(i, j)}=\operatorname{diag}\left(\mathbf{I}, \mathbf{I}, \ldots, \mathbf{I}+\mathbf{P}^{j}, \ldots, \mathbf{I}\right)
$$


where the matrix $\mathbf{I}+\mathbf{P}^{j}$ is in the $i$-th position of the array $\mathbf{D}^{(i, j)}$. Then, the matrix

$$
\mathbf{G}^{(i, j)}=\mathbf{H D}^{(i, j)} \mathbf{H}^{T}, \quad 0 \leq i<n^{\prime}, \quad 0 \leq j<\ell
$$

is an $m \times m$ matrix with $\delta$ 2's per row, corresponding to the $\delta$ lines that intersect the line of that row with the extra point not on the line, diagonal elements of $\rho$ and all other elements 0 or 1 . Note that the matrix $\mathbf{H D}^{(i, j)}$ contains an extra point in each row, each row corresponding to a line and a point not on the line. Conversely, if $\mathbf{H}$ is an $m \times n$ binary matrix which is an $m^{\prime} \times n^{\prime}$ array of CPMs of order $\ell$ satisfying these conditions, it represents a partial geometry $\operatorname{PaG}(\gamma, \rho, \delta)$. This is clear by construction. Thus, we have the following theorem that characterizes a binary array of CPMs to be a line-point adjacency matrix of a partial geometry.

Theorem 2. Let $\mathbf{H}$ be an $m \times n$ binary matrix written as an $m^{\prime} \times n^{\prime}$ array of CPMs of order $\ell$ whose associated matrices $\mathbf{G}^{(i, j)}$ of (3) satisfy the stated conditions. Then, $\mathbf{H}$ represents a partial geometry $\operatorname{Pa} G(\gamma, \rho, \delta)$.

The condition of the theorem does not allow zero matrix (ZM) of order $\ell$. The argument can be modified to accommodate this case although the result is more complicated and not presented here.

In Sections $\mathrm{V}$ and $\mathrm{VI}$, we will use Theorems 1 and 2 to construct two new classes of partial geometries whose line-point adjacency matrices are arrays of CPMs.

\section{B. Structural Properties}

Consider a partial geometry $\operatorname{PaG}(\gamma, \rho, \rho-1)$ as in the above Theorem 1. Notice that the line-point adjacency matrix $\mathbf{H}$ consists of $\gamma$ row-blocks of CPMs and $\rho$ column-blocks of CPMs of size $\gamma \times \gamma$. We first group the $\gamma \rho$ points into $\rho$ sets, denoted by $\mathbf{N}_{0}, \mathbf{N}_{1}, \ldots, \mathbf{N}_{\rho-1}$, each consisting of $\gamma$ points which correspond to $\gamma$ columns in a column-block of CPMs of the linepoint adjacency matrix $\mathbf{H}$. Since each row of a CPM has only one 1-entry, the $\rho$ points of a line $L$ are distributed in $\rho$ different sets, one point in each set. For $0 \leq k<\rho$, a point $v_{k}$ in the set $\mathbf{N}_{j}$ that is not on a line $L$ in $\operatorname{PaG}(\gamma, \rho, \rho-1)$ is not adjacent to the point on $L$ in the same set $\mathbf{N}_{j}$ but is adjacent to each of the other $\rho-1$ points on $L$ that are in the $\rho-1$ sets other than $\mathbf{N}_{j}$.

The $\gamma^{2}$ lines corresponding to the $\gamma^{2}$ rows of the line-point adjacency matrix $\mathbf{H}$ can be grouped into $\gamma$ bundles, denoted by $\mathbf{M}_{0}, \mathbf{M}_{1}, \ldots, \mathbf{M}_{\gamma-1}$, each consisting of $\gamma$ lines which correspond to 
the $\gamma$ rows in a row-block of CPMs in $\mathbf{H}$. Since any two rows in a row-block of CPMs in $\mathbf{H}$ do not have any position where they both have 1-entries, the $\gamma$ lines in each bundle $\mathbf{M}_{i}$ are parallel lines. Hence, each bundle $\mathbf{M}_{i}$ corresponds to a parallel bundle of lines in $\operatorname{PaG}(\gamma, \rho, \rho-1)$.

Let $\mathbf{H}_{0}, \mathbf{H}_{1}, \ldots, \mathbf{H}_{\gamma-1}$ denote the $\gamma$ row-blocks of $\mathbf{H}$. For $0 \leq i<\gamma$ and $0 \leq k<\gamma$, let $\mathbf{h}_{i, k}=\left(\mathbf{h}_{i, k, 0}, \mathbf{h}_{i, k, 1}, \ldots, \mathbf{h}_{i, k, \rho-1}\right)$ be the $k$-th row in the $i$-th row-block $\mathbf{H}_{i}$ which consists of $\rho$ sections, $\mathbf{h}_{i, k, 0}, \mathbf{h}_{i, k, 1}, \ldots, \mathbf{h}_{i, k, \rho-1}$, each consisting of $\gamma$ components. Each section is the $k$-th row of a CPM in $\mathbf{H}_{i}$. If we cyclically shift all the $\rho$ sections of $\mathbf{h}_{i, k}$ simultaneously one place to the right within the sections, we obtain the $(k+1)$-th row $\mathbf{h}_{i, k+1}$ of $\mathbf{H}_{i}$ which also consists of $\rho$ sections and each section is the $(k+1)$-th row of a CPM in $\mathbf{H}_{i}$. The above cyclic-shift within each section of $\mathbf{h}_{i, k}$ is referred to as section-wise cyclic-shift of the row $\mathbf{h}_{i, k}$. For $k=\gamma-1$, the section-wise cyclic-shift of $\mathbf{h}_{i, \gamma-1}$ results in the top row $\mathbf{h}_{i, 0}$ of $\mathbf{H}_{i}$. Consequently, all the rows of the $i$-th row-block $\mathbf{H}_{i}$ can be obtained by section-wise cyclically shifting the top row $\mathbf{h}_{i, 0}$ of $\mathbf{H}_{i} \gamma-1$ times.

Let $\mathbf{H}_{0}^{*}$ be an $\gamma \times \gamma \rho$ matrix over $\mathrm{GF}(2)$ which consists of the top rows $\mathbf{h}_{0,0}, \mathbf{h}_{1,0}, \ldots, \mathbf{h}_{\gamma-1,0}$ of the $\gamma$ row-blocks $\mathbf{H}_{0}, \mathbf{H}_{1}, \ldots, \mathbf{H}_{\gamma-1}$ of the line-point adjacency matrix $\mathbf{H}$ of $\operatorname{PaG}(\gamma, \rho, \rho-1)$. Then, it follows from the section-wise cyclic structure of $\mathbf{H}$, the entire array $\mathbf{H}$ can be obtained by section-wise cyclically shifting $\mathbf{H}_{0}^{\star} \gamma-1$ times. This structural property is referred to as $Q C$ (or section-wise cyclic) structure. The QC-structure of $\mathbf{H}$ implies that the incidence vectors of the $\gamma^{2}$ lines in the partial geometry $\operatorname{PaG}(\gamma, \rho, \rho-1)$ which correspond to the $\gamma^{2}$ rows of $\mathbf{H}$ can be generated by the incidence vectors of the $\gamma$ lines which correspond to the $\gamma$ rows in $\mathbf{H}_{0}^{*}$. That is to say that the lines corresponding to the rows of $\mathbf{H}_{0}^{*}$ and the points on these lines specify the partial geometry $\operatorname{PaG}(\gamma, \rho, \rho-1)$.

Due to the QC-structure of $\mathbf{H}$, we call the partial geometry $\operatorname{PaG}(\gamma, \rho, \rho-1)$ a quasi-cyclic partial geometry, denoted by $\mathrm{QC}-\mathrm{PaG}(\gamma, \rho, \rho-1)$.

In the following, we give an example to demonstrate that a $\mathrm{QC}-\mathrm{PaG}$ does exist.

Example 1. Consider the $3 \times 3$ array $\mathbf{H}$ of CPMs of size $3 \times 3$ given in the equation below: 


$$
\mathbf{H}=\left[\begin{array}{lllllllll}
1 & 0 & 0 & 1 & 0 & 0 & 1 & 0 & 0 \\
0 & 1 & 0 & 0 & 1 & 0 & 0 & 1 & 0 \\
0 & 0 & 1 & 0 & 0 & 1 & 0 & 0 & 1 \\
1 & 0 & 0 & 0 & 1 & 0 & 0 & 0 & 1 \\
0 & 1 & 0 & 0 & 0 & 1 & 1 & 0 & 0 \\
0 & 0 & 1 & 1 & 0 & 0 & 0 & 1 & 0 \\
1 & 0 & 0 & 0 & 0 & 1 & 0 & 1 & 0 \\
0 & 1 & 0 & 1 & 0 & 0 & 0 & 0 & 1 \\
0 & 0 & 1 & 0 & 1 & 0 & 1 & 0 & 0
\end{array}\right] .
$$

It is a $9 \times 9$ matrix over $G F(2)$. By checking, we find that it satisfies the RC-constraint. Hence, it follows from Theorem $\square$ that $\mathbf{H}$ is a line-point adjacency matrix of a $\mathrm{PaG}(3,3,2)$ with 9 points, 9 lines and connection number 2. Each line consists of 3 points and each point is on 3 lines. The lines in $\operatorname{PaG}(3,3,2)$ can be partitioned into 3 parallel bundles, each consisting of 3 parallel lines. Suppose we use $v_{0}, v_{1}, v_{2}, v_{3}, v_{4}, v_{5}, v_{6}, v_{7}, v_{8}$ to denote the 9 points in $\operatorname{Pa}(3,3,2)$ which correspond to the 9 columns of $\mathbf{H}$. Then, the 9 lines in $\operatorname{PaG}(3,3,2)$ are:

$$
\begin{aligned}
& L_{0}=\left\{v_{0}, v_{3}, v_{6}\right\}, \\
& L_{1}=\left\{v_{1}, v_{4}, v_{7}\right\}, \\
& L_{2}=\left\{v_{2}, v_{5}, v_{8}\right\}, \\
& L_{3}=\left\{v_{0}, v_{4}, v_{8}\right\}, \\
& L_{4}=\left\{v_{1}, v_{5}, v_{6}\right\}, \\
& L_{5}=\left\{v_{2}, v_{3}, v_{7}\right\}, \\
& L_{6}=\left\{v_{0}, v_{5}, v_{7}\right\}, \\
& L_{7}=\left\{v_{1}, v_{3}, v_{8}\right\}, \\
& L_{8}=\left\{v_{2}, v_{4}, v_{6}\right\} .
\end{aligned}
$$

Group the points in $\operatorname{PaG}(3,3,2)$ into 3 disjoint sets, $\mathbf{N}_{0}, \mathbf{N}_{1}$ and $\mathbf{N}_{2}$, each consisting of 3 points. For $0 \leq j<3$, the 3 points in $\mathbf{N}_{j}$ correspond to the 3 columns in the $j$-th columnblock of $\mathbf{H}$. Then, $\mathbf{N}_{0}=\left\{v_{0}, v_{1}, v_{2}\right\}, \mathbf{N}_{1}=\left\{v_{3}, v_{4}, v_{5}\right\}$ and $\mathbf{N}_{2}=\left\{v_{6}, v_{7}, v_{8}\right\}$. Consider the line $L_{3}=\left\{v_{0}, v_{4}, v_{8}\right\}$. The point $v_{5}$ is not on $L_{3}$. By checking, we find that this point is adjacent to 
the points $v_{0}$ and $v_{8}$. Notice that the point $v_{5}$ is in the set $\mathbf{N}_{1}$ but its two adjacent points $v_{0}$ and $v_{8}$ are in two separate sets $\mathbf{N}_{0}$ and $\mathbf{N}_{2}$. The graphical representation of $\operatorname{PaG}(3,3,2)$ is shown in Fig. 1 and the Tanner graph associated to the line-point adjacency matrix $\mathbf{H}$ is shown in Fig. 2. The girth of the graph is 6 .

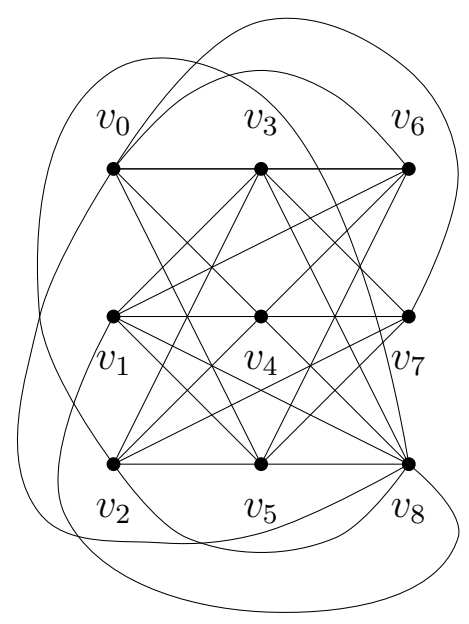

Fig. 1. A graphical representation of the partial geometry $\operatorname{PaG}(3,3,2)$ specified by its line-point adjacency matrix given by (4) in Example 1

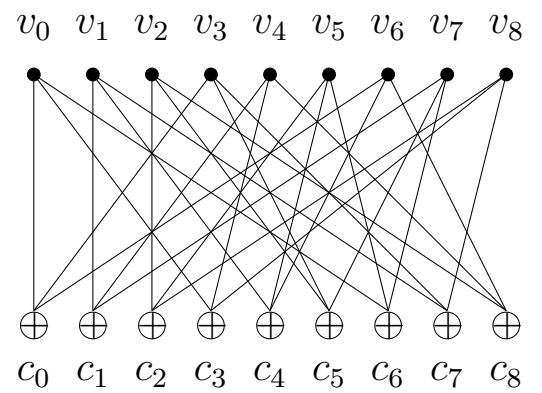

Fig. 2. The Tanner graph associated with the line-point adjacency matrix of $\operatorname{PaG}(3,3,2)$ given by (4) in Example 1

\section{Protograph Representation of A QC-PAG}

As shown in Section [I] a partial geometry with $n$ points and $m$ lines can be represented by a bipartite graph with $n$ VNs which represent the $n$ points of the geometry and $m$ CNs which 
represent the $m$ lines of the geometry. A VN $v_{j}$ is connected to a $\mathrm{CN} c_{i}$ by an edge $(i, j)$ if and only if the point represented by the $\mathrm{VN} v_{j}$ is on the line represented by the $\mathrm{CN} c_{i}$. For a partial geometry with a large set of points and a large set of lines, the bipartite graph representation of the geometry would be very large and complex. However, the QC-PaG $(\gamma, \rho, \rho-1)$ characterized by Theorem 1 can be effectively represented by a much smaller bipartite graph based on its QC-structure.

Recall that the line-point adjacency matrix $\mathbf{H}$ of the $\mathrm{QC}-\operatorname{PaG}(\gamma, \rho, \rho-1)$ is a binary $\gamma \times \rho$ array of CPMs of order $\gamma$ of the following form:

$$
\begin{aligned}
& \mathbf{H}=\left[\begin{array}{c}
\mathbf{H}_{0} \\
\mathbf{H}_{1} \\
\vdots \\
\mathbf{H}_{\gamma-1}
\end{array}\right] \\
& =\left[\begin{array}{cccc}
\mathbf{A}_{0,0} & \mathbf{A}_{0,1} & \cdots & \mathbf{A}_{0, \rho-1} \\
\mathbf{A}_{1,0} & \mathbf{A}_{1,1} & \cdots & \mathbf{A}_{1, \rho-1} \\
\vdots & \vdots & \cdots & \vdots \\
\mathbf{A}_{\gamma-1,0} & \mathbf{A}_{\gamma-1,1} & \cdots & \mathbf{A}_{\gamma-1, \rho-1}
\end{array}\right]
\end{aligned}
$$

The array $\mathbf{H}=\left[\mathbf{A}_{i, j}\right]_{0 \leq i<\gamma, 0 \leq j<\rho}$ consists of $\rho$ column-blocks of CPMs, denoted by $\mathbf{N}_{0}, \mathbf{N}_{1}, \ldots$, $\mathbf{N}_{\rho-1}$, and $\gamma$ row-blocks of CPMs, denoted by $\mathbf{H}_{0}, \mathbf{H}_{1}, \ldots, \mathbf{H}_{\gamma-1}$. For $0 \leq i<\gamma$ and $0 \leq j<\rho$, each column-block $\mathbf{N}_{j}$ consists of $\gamma$ consecutive columns of $\mathbf{H}$ and each row-block $\mathbf{H}_{i}$ consists of $\gamma$ consecutive rows of $\mathbf{H}$. The $\gamma$ columns of the $j$-th column-block $\mathbf{N}_{j}$ correspond to $\gamma$ points of the QC-PaG $(\gamma, \rho, \rho-1)$ and the $\gamma$ rows of the $i$-th row-block $\mathbf{H}_{i}$ correspond to $\gamma$ lines of the $\mathrm{QC}-\mathrm{PaG}(\gamma, \rho, \rho-1)$.

For $0 \leq j<\rho$, let $\Phi_{j}$ be the set of $\gamma$ points which correspond to the $\gamma$ columns of the $j$-th column-block $\mathbf{N}_{j}$ of $\mathbf{H}$. For $0 \leq i<\gamma$, let $\Omega_{i}$ be the set of lines which correspond to the $\gamma$ rows of the $i$-th row-block $\mathbf{H}_{i}$ of $\mathbf{H}$. In forming the Tanner graph $\mathcal{G}_{\mathrm{PaG}}$ of the $\mathrm{QC}-\mathrm{PaG}(\gamma, \rho, \rho-1)$, the $\gamma$ points in $\Phi_{j}$ are represented by $\gamma$ VNs and the $\gamma$ lines in $\Omega_{i}$ are represented by $\gamma$ CNs. Hereafter, we use points and VNs interchangeably, lines and CNs interchangeably. The $\gamma$ VNs in $\Phi_{j}$ are called type- $j$ VNs and the $\gamma$ CNs in $\Omega_{i}$ are called type- $i$ CNs. From the QC-structure of $\mathbf{H}$, we see that a type- $j \mathrm{VN}$ can only be connected to a type- $i \mathrm{CN}$ and vise versa. In forming the Tanner graph $\mathcal{G}_{\mathrm{PaG}}$ of the QC-PaG $(\gamma, \rho, \rho-1)$, the $\gamma$ type- $j$ VNs in $\Phi_{j}$ are connected to the $\gamma$ type- $i$ CNs in $\Omega_{i}$ based on the $\gamma$ 1-entires in the CPM $\mathbf{A}_{i, j}$ and vise versa. If we label the 
columns and rows of a CPM $\mathbf{A}_{i, j}$ in $\mathbf{H}$ from 0 to $\gamma-1$, then $\mathbf{A}_{i, j}$ is uniquely specified by the location of the single 1-entry of its top row, called the generator. If the single 1-entry of the top row of $\mathbf{A}_{i, j}$ locates at the position $k_{i, j}, 0 \leq k_{i, j}<\gamma$, then we use $\left(k_{i, j}\right)$ to specify the CPM $\mathbf{A}_{i, j}$.

With all the terms defined above, we now construct a bipartite graph, denoted by $\mathcal{G}_{\text {proto,PaG }}$, with $\rho$ VNs and $\gamma \mathrm{CNs}$ as shown in Fig. 3. The $\rho$ VNs of $\mathcal{G}_{\text {proto, PaG }}$ represent the $\rho$ clusters $\Phi_{0}, \Phi_{1}, \ldots, \Phi_{\rho-1}$ of $\mathrm{VNs}$ in $\mathcal{G}_{\mathrm{PaG}}$ and the $\gamma \mathrm{CNs}$ in $\mathcal{G}_{\text {proto,PaG }}$ represent the $\gamma$ clusters $\Omega_{0}, \Omega_{1}, \ldots, \Omega_{\gamma-1}$ of $\mathrm{CNs}$ in $\mathcal{G}_{\mathrm{PaG}}$. In $\mathcal{G}_{\text {proto,PaG }}$, the $\mathrm{VN} \Phi_{j}$ is connected to the $\mathrm{CN} \Omega_{i}$ by an edge labeled by $\left(k_{i, j}\right)$ which is the location of the single 1-entry of the generator (or top row) of the CPM $\mathbf{A}_{i, j}$ in the line-point adjacency matrix $\mathbf{H}$ of the QC-PaG $(\gamma, \rho, \rho-1)$. The bipartite graph $\mathcal{G}_{\text {proto,PaG }}$ contains all the structural information of the $\mathrm{QC}-\mathrm{PaG}(\gamma, \rho, \rho-1)$. The size of the bipartite graph $\mathcal{G}_{\text {proto,PaG }}$ is smaller than the size of the bipartite graph $\mathcal{G}_{\mathrm{PaG}}$ by a factor $\gamma$. This bipartite graph $\mathcal{G}_{\text {proto,PaG }}$ is called the protograph of the QC-PaG $(\gamma, \rho, \rho-1)$. Basically, the protograph $\mathcal{G}_{\text {proto,PaG }}$ of the $\mathrm{QC}-\mathrm{PaG}(\gamma, \rho, \rho-1)$ consists of $\rho$ super-VNs, $\Phi_{0}, \Phi_{1}, \ldots, \Phi_{\rho-1}$, and $\gamma$ super-CNs, $\Omega_{0}, \Omega_{1}, \ldots, \Omega_{\gamma-1}$. The Tanner graph $\mathcal{G}_{\mathrm{PaG}}$ of the QC-PaG $(\gamma, \rho, \rho-1)$ is simply an expansion of the protograph $\mathcal{G}_{\text {proto,PaG }}$ of the $\mathrm{QC}-\mathrm{PaG}(\gamma, \rho, \rho-1)$.

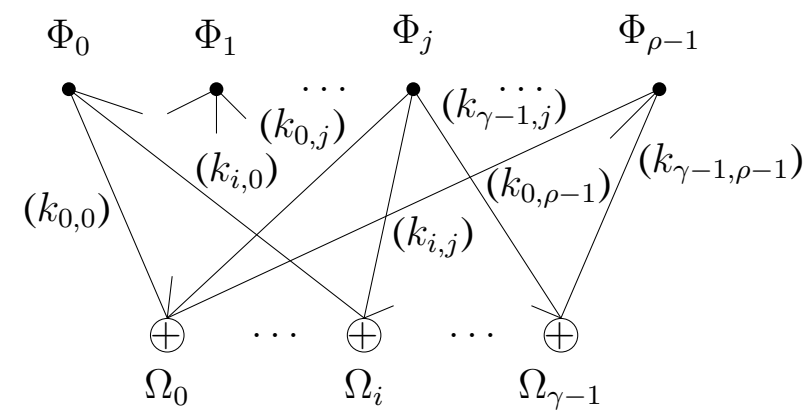

Fig. 3. The protograph of the QC-PaG $(\gamma, \rho, \rho-1)$.

Example 2. Consider the $Q C-P a G(3,3,2)$ given in Example 1 We group the 9 points of the geometry into three disjoint sets, $\Phi_{0}=\left\{v_{0}, v_{1}, v_{2}\right\}, \Phi_{1}=\left\{v_{3}, v_{4}, v_{5}\right\}, \Phi_{2}=\left\{v_{6}, v_{7}, v_{8}\right\}$, and the 9 lines of the geometry into three disjoint sets, $\Omega_{0}=\left\{L_{0}, L_{1}, L_{2}\right\}, \Omega_{1}=\left\{L_{3}, L_{4}, L_{5}\right\}$, $\Omega_{2}=\left\{L_{6}, L_{7}, L_{8}\right\}$. Using $\Phi_{0}, \Phi_{1}, \Phi_{2}$ as the super-VNs and $\Omega_{0}, \Omega_{1}, \Omega_{2}$ as the super-CNs, we form the protograph $\mathcal{G}_{\text {proto,PaG }}$ of the $Q C-P a G(3,3,2)$ as shown in Fig. 4 Each edge is labeled by the position of the single 1-entry of the generator of each CPM which specifies the connections between the constituent VNs of a super-VN $\Phi_{j}$ in $\mathcal{G}_{\text {proto,PaG }}$ and the constituent CNs of a super-CN $\Omega_{i}$ in $\mathcal{G}_{\text {proto,PaG }}$. 


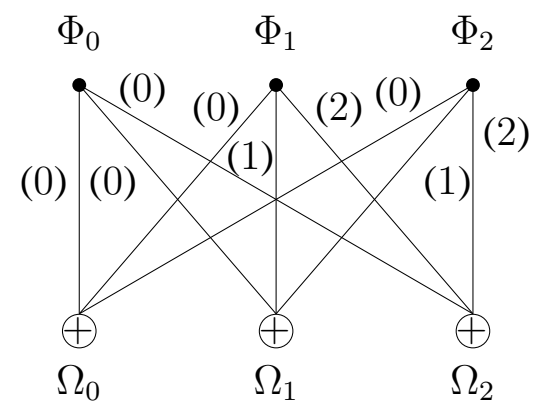

Fig. 4. The protograph of the $\mathrm{QC}-\mathrm{PaG}(3,3,2)$ given in Example 2

LDPC codes can be constructed based on relatively small protographs [1], [13], [14], [47]. These LDPC codes are called protograph LDPC codes. In constructing such a code, we take $\gamma$ copies of a chosen protograph. Then, we permute the edges of the individual copies and connect the copies into a large Tanner graph. The null space of the adjacency matrix of the Tanner graph gives a protograph LDPC code. Constructions of protograph LDPC codes in most of the literature are pseudo-random and require computer aid to perform the connections of the copies of the chosen protograph.

From the graph point of view, the QC-LDPC code constructed based on the QC-PaG $(\gamma, \rho, \rho-$ 1) may be regarded as a protograph QC-LDPC code. Based on the description of the QC$\operatorname{PaG}(\gamma, \rho, \rho-1)$, we first construct its protograph $\mathcal{G}_{\text {proto,PaG }}$. Then, we take $\gamma$ copies of $\mathcal{G}_{\text {proto,PaG }}$, permute the edges of the copies and connect the copies to form the Tanner graph $\mathcal{G}_{\mathrm{PaG}}$ of the QC-PaG $(\gamma, \rho, \rho-1)$. The null space of the adjacency matrix of the Tanner graph $\mathcal{G}_{\mathrm{PaG}}$ of the QC-PaG $(\gamma, \rho, \rho-1)$ gives a protograph QC-LDPC code. In Sections $\nabla$ and VI, we will present two classes of QC-PaGs. The protograph of a QC-PaG from each of these two classes can be constructed directly from the description of its base matrix.

\section{A Class of QC-PaGs Constructed Based on Prime Fields}

In this section, we present a class of QC-PaGs based on prime fields whose line-point adjacency matrices are arrays of CPMs.

\section{A. Construction}

Let $p$ be a prime and $\operatorname{GF}(p)$ be a prime field which consists of the following $p$ elements: $0,1,2, \ldots, p-1$. We represent each element $i$ in $\mathrm{GF}(p)$ by a $p \times p \mathrm{CPM}$, denoted by $\mathbf{Q}(i)$, with columns and rows labeled from 0 to $p-1$, whose generator has its single 1-component at the 
location $i$. This representation is one-to-one. For $i=0, \mathbf{Q}(0)$ is a $p \times p$ identity matrix. $\mathbf{Q}(i)$ is referred to as the CPM-dispersion of the element $i$ in $\operatorname{GF}(p)$.

Form the following $p \times p$ matrix $\mathbf{B}_{p}$ over $\operatorname{GF}(p)$ with columns and rows labeled from 0 to $p-1$ :

$$
\mathbf{B}_{p}=\left[\begin{array}{cccc}
0 \cdot 0 & 0 \cdot 1 & \cdots & 0 \cdot(p-1) \\
1 \cdot 0 & 1 \cdot 1 & \cdots & 1 \cdot(p-1) \\
2 \cdot 0 & 2 \cdot 1 & \cdots & 2 \cdot(p-1) \\
\vdots & \vdots & \cdots & \vdots \\
(p-1) \cdot 0 & (p-1) \cdot 1 & \cdots & (p-1) \cdot(p-1)
\end{array}\right]
$$

i.e., $\mathbf{B}_{p}=\left[b_{i j}\right]_{0 \leq i, j<p-1}$ with $b_{i j}=i j$, where the multiplication of two elements in $\operatorname{GF}(p)$ is carried out under modulo $p$.

The matrix $\mathbf{B}_{p}$ has the following structural properties:

1) All the entries in the 0-th row and column are zeros;

2) All the entries in any row or column, other than the 0-th row and column, are different and contain all the elements of $\operatorname{GF}(p)$;

3) Two different rows or columns have the 0 element of $\operatorname{GF}(p)$ in common at the 0 -th row or column and differ in all the other $p-1$ positions;

4) The $i$-th column of $\mathbf{B}_{p}$ is identical to the transpose of $i$-th row of $\mathbf{B}_{p}$ for $0 \leq i<p$. The last property implies that the transpose $\mathbf{B}_{p}^{T}$ of $\mathbf{B}_{p}$ is identical to $\mathbf{B}_{p}$, i.e., $\mathbf{B}_{p}{ }^{T}=\mathbf{B}_{p}$.

If each entry in $\mathbf{B}_{p}$ is dispersed into its corresponding $p \times p \mathbf{C P M}$, we obtain a $p \times p$ array $\mathbf{H}_{p}$ of CPMs of size $p \times p$. It is a $p^{2} \times p^{2}$ matrix over $\mathrm{GF}(2)$. Each row has weight $p$ and each column has weight $p$. Based on the structural properties of $\mathbf{B}_{p}$, we can readily see that $\mathbf{H}_{p}$ satisfies the RC-constraint. Hence, it follows from Theorem 1 that $\mathbf{H}_{p}$ is the line-point adjacency matrix of a $\mathrm{QC}_{-} \mathrm{PaG}_{p}(p, p, p-1)$ with columns corresponding to the points and rows corresponding to the lines in the $\mathrm{QC}-\mathrm{PaG}_{p}(p, p, p-1)$. Equally, Theorem 2 could have been applied. The partial geometry $\mathrm{QC}-\mathrm{PaG}_{p}(p, p, p-1)$ consists of $n=p^{2}$ points and $m=p^{2}$ lines. Each line in the QC- $\mathrm{PaG}_{p}(p, p, p-1)$ consists of $p$ points and each point is on $p$ lines. A point $v$ that is not on a line $L$ is connected to $p-1$ points on $L$ by lines, i.e., the connection number of a point in the $\mathrm{QC}-\mathrm{PaG}_{p}(p, p, p-1)$ is $p-1$. The array $\mathbf{H}_{p}$ is called the CPM-dispersion of $\mathbf{B}_{p}$ and $\mathbf{B}_{p}$ is 
called the base matrix for the construction of the $\mathrm{QC}-\mathrm{PaG}_{p}(p, p, p-1)$. The subscript " $p$ " of the QC-PaG ${ }_{p}(p, p, p-1)$ stands for "prime".

As an array of CPMs, $\mathbf{H}_{p}$ consists of $p$ row-blocks of CPMs and $p$ column-block of CPMs. Two different rows in a row-block of $\mathbf{H}_{p}$ have no position where they both have 1-entries. Hence, the $p$ rows in each row-block correspond to $p$ parallel lines in the $\mathrm{QC}_{-}-\mathrm{PaG}_{p}(p, p, p-1)$. Therefore, the $\mathrm{QC}-\mathrm{PaG}_{p}(p, p, p-1)$ consists of $p$ parallel bundles, each consisting of $p$ parallel lines. Let $\mathbf{M}_{0}, \mathbf{M}_{1}, \ldots, \mathbf{M}_{p-1}$ denote the $p$ parallel bundles of lines in the $\mathrm{QC}_{-} \mathrm{PaG}_{p}(p, p, p-1)$. For $0 \leq i<p$, the $p$ lines in the $i$-th parallel bundle $\mathbf{M}_{i}$ correspond to the $p$ rows in the $i$-th row-block of $\mathbf{H}_{p}$. The $p$ lines in each parallel bundle contain all the $p^{2}$ points in the QC$\operatorname{PaG}_{p}(p, p, p-1)$. This partial geometry is a net.

The $p$ rows in $\mathbf{H}_{p}$ that have 1-entries at the $j$-th position are in $p$ different row-blocks. These rows correspond to $p$ lines in an intersecting bundle of lines that intersect at the point $v_{j}$ corresponding to the $j$-th column of $\mathbf{H}_{p}$. The $p$ lines in an intersecting bundle are in $p$ different parallel bundles of the $\mathrm{QC}-\mathrm{PaG}_{p}(p, p, p-1)$.

The $p^{2}$ points in the $\mathrm{QC}_{-} \mathrm{PaG}_{p}(p, p, p-1)$ can be divided into $p$ sets, $\mathbf{N}_{0}, \mathbf{N}_{1}, \ldots, \mathbf{N}_{p-1}$, each consisting of $p$ points. For $0 \leq j<p$, the $p$ points in the $j$-th set $\mathbf{N}_{j}$ correspond to the $p$ columns of the $j$-th column-block of $\mathbf{H}_{p}$. The $p$ points of a line $L$ are distributed in $p$ different sets, one and only one point in each set. A point $v$ in the set $\mathbf{N}_{j}$ that is not on a line $L$ is adjacent to one and only one point on $L$ in each set other than $\mathbf{N}_{j}$.

It follows from the results on the structural properties given in [21] that the Tanner graph, denoted by $\mathcal{G}_{p, \mathrm{PaG}}$, of the $\mathrm{QC}^{-\mathrm{PaG}_{p}}(p, p, p-1)$ has girth 6 and contains $p^{3}(p-1)^{2}(p-2) / 6$ cycles of length 6 .

The protograph, denoted by $\mathcal{G}_{p, \text { proto,PaG }}$ of the $\mathrm{QC}^{-\mathrm{PaG}_{p}}(p, p, p-1)$ can be constructed directly from the base matrix $\mathbf{B}_{p}$ given by (6). It consists of $p$ VNs and $p$ CNs, labeled by $\Phi_{0}, \Phi_{1}, \ldots, \Phi_{p-1}$ and $\Omega_{0}, \Omega_{1}, \ldots, \Omega_{p-1}$, respectively. The $p$ VNs and $p$ CNs of the protograph $\mathcal{G}_{p \text {,proto,PaG }}$ correspond to the $p$ columns and $p$ rows of the base matrix $\mathbf{B}_{p}$ of the $\mathrm{QC}^{-} \mathrm{PaG}_{p}(p, p, p-1)$. Every $\mathrm{VN}$ is connected to every $\mathrm{CN}$. The edge $(j, i)$ connecting the $\mathrm{VN} \Phi_{j}$ to the $\mathrm{CN} \Omega_{i}$ is labeled by the integer $i j$ modulo $p$.

If we remove the first column and the first row from the base matrix $\mathbf{B}_{p}$, we obtain a Latin square of order $p-1$. The use of Latin squares for constructing LDPC codes was considered in [25], [54]. 
Example 3. Let $p=3$ and let $G F(3)$ be the field to construct a $Q C-P a G_{p}(3,3,2)$. Using (6), we find the base matrix for constructing the line-point adjacency matrix $\mathbf{H}_{p}$ of the $Q C$-PaG $G_{p}(3,3,2)$ is

$$
\mathbf{B}_{p}=\left[\begin{array}{lll}
0 & 0 & 0 \\
0 & 1 & 2 \\
0 & 2 & 1
\end{array}\right] .
$$

The $3 \times 3$ CPM-dispersion $\mathbf{H}_{p}$ of $\mathbf{B}_{p}$ is

$$
\mathbf{H}_{p}=\left[\begin{array}{lllllllll}
1 & 0 & 0 & 1 & 0 & 0 & 1 & 0 & 0 \\
0 & 1 & 0 & 0 & 1 & 0 & 0 & 1 & 0 \\
0 & 0 & 1 & 0 & 0 & 1 & 0 & 0 & 1 \\
1 & 0 & 0 & 0 & 1 & 0 & 0 & 0 & 1 \\
0 & 1 & 0 & 0 & 0 & 1 & 1 & 0 & 0 \\
0 & 0 & 1 & 1 & 0 & 0 & 0 & 1 & 0 \\
1 & 0 & 0 & 0 & 0 & 1 & 0 & 1 & 0 \\
0 & 1 & 0 & 1 & 0 & 0 & 0 & 0 & 1 \\
0 & 0 & 1 & 0 & 1 & 0 & 1 & 0 & 0
\end{array}\right],
$$

which is exactly the line-point adjacency matrix of the $P a G(3,3,2)$ given by (4) in Example 2 The Tanner graph and the protograph of this partial geometry are shown in Fig. 2 and Fig. 4 respectively.

\section{B. Subgeometries}

Let $\tau$ be a positive integer less than $p-1$. Suppose we delete $\tau$ column-blocks from $\mathbf{H}_{p}$. We obtain a $p \times(p-\tau)$ subarray, denoted by $\mathbf{H}_{p}(p, p-\tau)$, of $\mathbf{H}_{p}$. It follows from the structural property developed above that $\mathbf{H}_{p}(p, p-\tau)$ is the line-point adjacency matrix of a QC-PaG $p(p, p-$ $\tau, p-\tau-1)$ which has $p(p-\tau)$ points and $p^{2}$ lines, each point on $p$ lines and each line consisting of $p-\tau$ points. The connection number of a point in the $\mathrm{QC}-\mathrm{PaG}_{p}(p, p-\tau, p-\tau-1)$ is $p-\tau-1$. The $\mathrm{QC}-\mathrm{PaG}_{p}(p, p-\tau, p-\tau-1)$ is a subgeometry of the $\mathrm{QC}-\mathrm{PaG}_{p}(p, p, p-1)$. Its Tanner graph (or protograph) is a subgraph of the Tanner graph (or protograph) of the $\mathrm{QC}-\mathrm{PaG}_{p}(p, p, p-1$ ).

Therefore, for a given prime field, we can construct a family of QC-PaGs. 
C. QC-LDPC Codes on the $Q C-P a G_{p}(p, p, p-1)$

For $1 \leq k, r \leq p$, let $\mathbf{B}_{p}(k, r)$ be a $k \times r$ submatrix of the matrix $\mathbf{B}_{p}$ of (6). CPM-dispersing the entries of $\mathbf{B}_{p}(k, r)$, we obtain a $k \times r$ array $\mathbf{H}_{p}(k, r)$ of CPMs of size $p \times p$ which is a subarray of $\mathbf{H}_{p}$. The array $\mathbf{H}_{p}(k, r)$ is a $k p \times r p$ matrix with column and row weights, $k$ and $r$, respectively. The null space of $\mathbf{H}_{p}(k, r)$ gives a $(k, r)$-biregular QC-LDPC code, denoted by $C_{p, q c}(k, r)$, of length $r p$ with rate at least $(r-k) / r$. Therefore, for a given prime field, a family of QC-LDPC codes of various lengths and rates can be constructed. The Tanner graph of $C_{p, q c}(k, r)$ has girth of at least 6.

Express $\mathbf{B}_{p}(k, r)$ as $\mathbf{B}_{p}(k, r)=\left[b_{i, j}\right]_{0 \leq i<k, 0 \leq j<r}$. Suppose an entry in $\mathbf{B}_{p}(k, r)$ is replaced by the zero element of $\operatorname{GF}(p)$. In the CPM-dispersion $\mathbf{H}_{p}(k, r)$ of $\mathbf{B}_{p}(k, r)$, this replacement results in replacing a $p \times p \mathrm{CPM}$ in $\mathbf{H}_{p}(k, r)$ by a $p \times p$ zero matrix (ZM) which is referred to as masking [11], [12], [42], [53]. Let $\lambda$ be a nonnegative integer less than the number of total nonzero entries in $\mathbf{B}_{p}(k, r)$. The replacement of $\lambda$ nonzero entries in $\mathbf{B}_{p}(k, r)$ by $\lambda$ zeros amounts to replacing $\lambda$ CPMs by $\lambda$ ZMs at the locations in $\mathbf{H}_{p}(k, r)$ corresponding to the locations of the $\lambda$ entries in $\mathbf{B}_{p}(k, r)$ which are replaced by zeros. Masking $\lambda \mathbf{C P M s}$ in $\mathbf{H}_{p}(k, r)$ amounts to removing $\lambda p$ edges from the Tanner graph $\mathcal{G}_{p}(k, r)$ associated with $\mathbf{H}_{p}(k, r)$. Removing these edges in $\mathcal{G}_{p}(k, r)$ may break many short cycles in $\mathcal{G}_{p}(k, r)$. As a result, the resultant Tanner graph $\mathcal{G}_{p, \text { mask }}(k, r)$ may have a much smaller number of short cycles, or a larger girth, or both. The subscript "mask" stands for "masking". In choosing the entries in $\mathbf{B}_{p}(k, r)$ to be masked, we have to avoid disconnecting the Tanner graph of $\mathbf{H}_{p}(k, r)$.

The operation of masking $\mathbf{B}_{p}(k, r)=\left[b_{i, j}\right]_{0 \leq i<k, 0 \leq j<r}$ can be modeled mathematically. Let $\mathbf{Z}(k, r)=\left[z_{i, j}\right]_{0 \leq i<k, 0 \leq j<r}$ be a $k \times r$ matrix with the zero element and unit element of $\operatorname{GF}(p)$ as entries. Define the following product of $\mathbf{Z}(k, r)$ and $\mathbf{B}_{p}(k, r): \mathbf{B}_{p \text {,mask }}(k, r)=\mathbf{Z}(k, r) \odot$ $\mathbf{B}_{p}(k, r)=\left[z_{i, j} b_{i, j}\right]_{0 \leq i<k, 0 \leq j<r}$ (the Hadamard product) where $z_{i, j} b_{i, j}=b_{i, j}$ if $z_{i, j}=1$ and $z_{i, j} b_{i, j}=0$ if $z_{i, j}=0$. In this matrix product operation, entries in $\mathbf{B}_{p}(k, r)$ at the locations corresponding to the locations of zero-entries in $\mathbf{Z}(k, r)$ are replaced (or masked) by 0's. The CPM-dispersion of $\mathbf{B}_{p \text {, mask }}(k, r)$ gives a $k \times r$ masked array $\mathbf{H}_{p \text {,mask }}(k, r)$ of CPMs and ZMs of size $p \times p$. We call $\mathbf{Z}(k, r)$ and $\mathbf{B}_{p \text {,mask }}(k, r)$ the masking matrix and the masked base matrix, respectively. The null space of $\mathbf{H}_{p \text {,mask }}(k, r)$ also gives a QC-LDPC code, denoted by $C_{p, q c, \text { mask }}(k, r)$.

Example 4. Consider the $127 \times 127$ base matrix $\mathbf{B}_{p}$ over GF(127) given in (6). It contains the 
following $4 \times 8$ submatrix $\mathbf{B}_{p}(4,8)$ (rows and columns chosen at random):

$$
\mathbf{B}_{p}(4,8)=\left[\begin{array}{cccccccc}
2 & 83 & 33 & 46 & 36 & 94 & 42 & 86 \\
109 & 15 & 84 & 94 & 57 & 43 & 3 & 115 \\
112 & 76 & 70 & 36 & 111 & 57 & 66 & 117 \\
31 & 80 & 67 & 78 & 50 & 60 & 16 & 63
\end{array}\right] .
$$

We design the following masking matrix:

$$
\mathbf{Z}(4,8)=\left[\begin{array}{llllllll}
1 & 0 & 1 & 0 & 1 & 1 & 1 & 1 \\
0 & 1 & 0 & 1 & 1 & 1 & 1 & 1 \\
1 & 1 & 1 & 1 & 1 & 0 & 1 & 0 \\
1 & 1 & 1 & 1 & 0 & 1 & 0 & 1
\end{array}\right]
$$

Masking $\mathbf{B}_{p}(4,8)$ with $\mathbf{Z}(4,8)$ gives the matrix $\mathbf{B}_{p, \text { mask }}(4,8)$. Replacing each nonzero entry in $\mathbf{B}_{p, \text { mask }}(4,8)$ by its corresponding $127 \times 127$ CPM and each 0-entry by a $127 \times 127$ ZM, we obtain a masked $4 \times 8$ array $\mathbf{H}_{p, \text { mask }}(4,8)$. It is a $508 \times 1016$ matrix with column and row weights 3 and 6, respectively. The null space of $\mathbf{H}_{p, \text { mask }}(4,8)$ gives a $(3,6)$-biregular binary $(1016,508)$ QC-LDPC code with rate 0.5. The Tanner graph of this code has girth 8 and contains 889 cycles of length 8. The bit and block error performances of the code decoded with 5, 10 and 50 iterations of the min-sum algorithm (MSA) [10] are shown in Fig. 5] We see that the code has a very low error-floor. Included in the same figure are the bit and block performances of an LDPC code constructed using the progressive edge growth (PEG) algorithm [19]. The PEG code is only decoded with 50 iterations of the MSA. We see that the code constructed based on GF(127) outperforms the PEG code below the bit error rate (BER) of $10^{-7}$.

As noted, a special case of the partial geometries is when $\delta=1$, the generalized quadrangles (GQs). In this case there are no triangles in the geometry and hence the associated graph has girth 8. There have been numerous studies on the use of GQs in coding theory (although none considered their trapping sets to our knowledge). The works include [2]-[4], [30], [31], [36], [38], [40], [51], [52]. Some comments on the codes from GQs derived from these papers are given. The paper of Liu et al [31] contains a very useful summary of the minimum distance bounds and rates of the various codes resulting from using GQs for code constructions. Among other things, that work established the following two important results:

1) A code whose parity-check matrix is the adjacency matrix of a generalized $d$-gon, for $n$ 


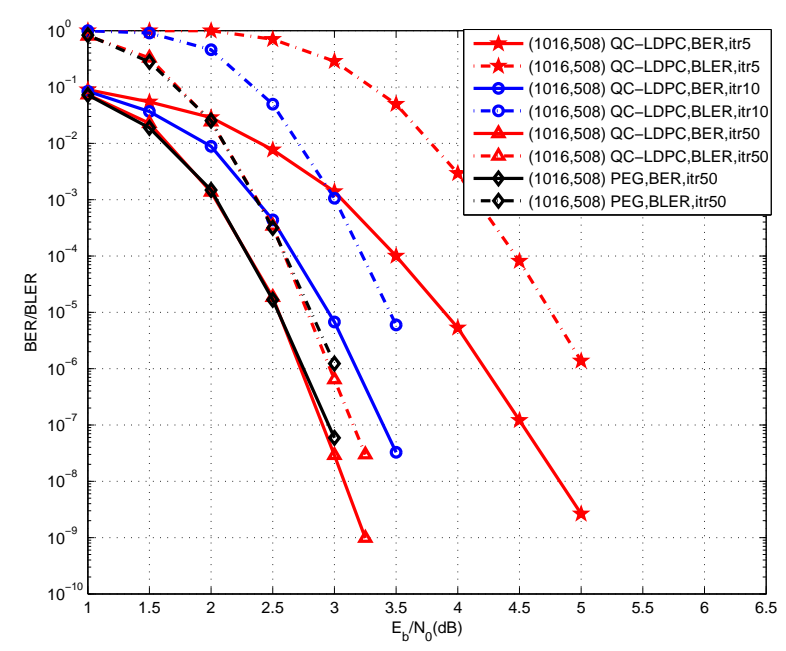

Fig. 5. Performance curves for the $(1016,508)$ QC-LDPC code and the PEG code given in Example 4

even, has only codewords of even weight.

2) A code whose parity check-matrix is the adjacency matrix of a GQ is quasi-cyclic.

This last point is important for code implementation. Its proof considers the transitivity of the automorphism group of the quadrangle which produces different sized circulant blocks for the different constructions. The reader is referred to [31] for details.

\section{A Class of QC-PaGs Constructed Based On Cyclic Subgroups of Finite FIELDS}

In this section, we present another class of QC-PaGs which are constructed based on the cyclic subgroups of finite fields.

\section{A. Construction}

Let $\operatorname{GF}(q)$ be finite field with $q$ elements where $q$ is a power of a prime. Let $t$ be a prime factor of $q-1$ and $q-1=c t$. If $\alpha$ is a primitive element of $\operatorname{GF}(q)$, then $\beta=\alpha^{c}$ is an element of order $t$ in $\operatorname{GF}(q)$. The set $\mathbf{S}=\left\{1, \beta, \beta^{2}, \ldots, \beta^{(t-1)}\right\}$ forms a cyclic subgroup of $\operatorname{GF}(q)$ of order $t$. 
Form the following $t \times t$ matrix:

$$
\mathbf{B}_{c}=\left[\begin{array}{ccccc}
1 & 1 & 1 & \cdots & 1 \\
1 & \beta & \beta^{2} & \cdots & \beta^{t-1} \\
1 & \beta^{2} & \left(\beta^{2}\right)^{2} & \cdots & \left(\beta^{2}\right)^{t-1} \\
\vdots & \vdots & \vdots & \cdots & \vdots \\
1 & \beta^{t-1} & \left(\beta^{t-1}\right)^{2} & \cdots & \left(\beta^{t-1}\right)^{t-1}
\end{array}\right]
$$

Express the above matrix in the form $\mathbf{B}_{c}=\left[\beta^{i j}\right]_{0 \leq i, j<t}$ whose $(i, j)$-th element is $\beta^{i j}$. The subscript " $c$ " stands for "cyclic group". All the entries of $\mathbf{B}_{c}$ are elements of the cyclic subgroup S. Let $\mathbf{P}$ denote the CPM of size $t \times t$ whose first (or the top) row is the $t$-tuple $(0,1,0, \cdots, 0)$ with the single 1-component at the position-1 (the positions are labeled from 0 to $t-1$ ). For $0 \leq \ell<t$, the $\ell$-th power of $\mathbf{P}$, denoted by $\mathbf{P}^{\ell}$ is a CPM of size $t \times t$ whose top row has its single 1-component at the position- $\ell$. Then, $\left\{\mathbf{I}, \mathbf{P}, \ldots, \mathbf{P}^{(t-1)}\right\}$ is a cyclic matrix group of order $t$ with $\mathbf{P}$ as a generator and $\mathbf{P}^{t}=\mathbf{P}^{0}=\mathbf{I}$.

For $0 \leq \ell<t$, we represent the element $\beta^{\ell}$ in the cyclic group $\mathbf{S}$ of $\operatorname{GF}(q)$ by the $\operatorname{CPM} \mathbf{P}^{\ell}$. This representation is one-to-one and $\mathbf{P}^{\ell}$ is the CPM-dispersion of $\beta^{\ell}$.

Let $\mathbf{H}_{c}=\mathbf{B}_{c}(\mathbf{P})$ denote the CPM-dispersion of the matrix $\mathbf{B}_{c}$, i.e., for $0 \leq i, j<t$, the entry $\beta^{i j}$ of $\mathbf{B}_{c}$ at the location $(i, j)$ is dispersed into the $\mathrm{CPM} \mathbf{P}^{l}$ where $\ell=(i j)$ modulo $t$. Then, $\mathbf{H}_{c}=\mathbf{B}_{c}(\mathbf{P})$ is a $t \times t$ array of CPMs of size $t \times t$. This array $\mathbf{H}_{c}=\mathbf{B}_{c}(\mathbf{P})$ is a $t^{2} \times t^{2}$ binary matrix which is the line-point adjacency matrix of the $\mathrm{QC}-\mathrm{PaG}_{c}(t, t, t-1)$. The result can be established using either Theorem 1 or Theorem 2 , but Theorem 2 is used for illustrative purposes. Notice that $\mathbf{P}^{T}=\mathbf{P}^{-1}$. Consider the product of $\mathbf{B}_{c}(\mathbf{P})$ and its transpose $\left[\mathbf{B}_{c}(\mathbf{P})\right]^{T}$. Express the product $\mathbf{B}_{c}(\mathbf{P})\left[\mathbf{B}_{c}(\mathbf{P})\right]^{T}$ as a $t \times t$ array of $t \times t$ matrices. Then, we have

$$
\mathbf{A}=\left[\mathbf{A}_{r, s}\right]_{0 \leq r, s<t}=\mathbf{B}_{c}(\mathbf{P})\left[\mathbf{B}_{c}(\mathbf{P})\right]^{T}
$$

For $0 \leq r, s<t$, the constituent matrix $\mathbf{A}_{r, s}$ of $\mathbf{A}$ is

$$
\begin{aligned}
\mathbf{A}_{r, s} & =\sum_{u=0}^{t-1} \mathbf{P}^{u j}\left(\mathbf{P}^{T}\right)^{u s} \\
& =\sum_{u=0}^{t-1} \mathbf{P}^{u(r-s)}=\left\{\begin{array}{r}
\mathbf{J} \text { if } r \neq s \\
\rho \mathbf{I} \text { if } r=s
\end{array}\right.
\end{aligned}
$$

where $\mathbf{J}$ is the $t \times t$ all ones matrix and $\mathbf{I}$ the $t \times t$ identity matrix. Suppose the $i$-th column-block 
of CPMs of $\mathbf{B}(\mathbf{P})$ is multiplied by $\mathbf{I}+\mathbf{P}^{j}$ to give $\mathbf{A}^{(i, j)}=\left(\mathbf{A}_{r, s}^{\prime}\right)$ where

$$
\mathbf{A}_{r, s}^{\prime}=\mathbf{A}_{r, s}+\mathbf{P}^{i(r-s)+j} .
$$

Thus, each CPM of each row-block of $\mathbf{B}_{c}(\mathbf{P})$ has a CPM added to it. For a non-diagonal circulant $(r \neq s)$, this means each row has $(t-1) 2$ 's with all remaining elements in the row being 0 or 1 . It follows from Theorem 2 that the array $\mathbf{H}_{c}=\mathbf{B}_{c}(\mathbf{P})$, as a $t^{2} \times t^{2}$ matrix, is the line-point adjacency matrix of a partial geometry $\mathrm{PaG}_{c}(t, t, t-1)$. The $\mathrm{PaG}_{c}(t, t, t-1)$ is a QC-PaG consisting of $n=t^{2}$ points and $m=t^{2}$ lines with connection number $\delta=t-1$. This partial geometry $\mathrm{PaG}_{c}(t, t, t-1)$ is also a net.

If we want to use Theorem 1 to prove $\mathbf{H}_{c}=\mathbf{B}_{c}(\mathbf{P})$ is the line-point adjacency matrix of the partial geometry $\mathrm{PaG}_{c}(t, t, t-1)$, we need to show that any $2 \times 2$ submatrix of $\mathbf{B}_{c}$ is nonsigular. This can be done easily. It follows from [55, Corollary 1] that $\mathbf{H}_{c}$ as a $t^{2} \times t^{2}$ matrix satisfies the RC-constraint. Consequently, it follows from Theorem 1 that $\mathbf{H}_{c}=\mathbf{B}_{c}(\mathbf{P})$ is the line-point adjacency matrix of the partial geometry $\mathrm{PaG}_{c}(t, t, t-1)$.

The above construction gives another class of QC-PaGs whose line-point adjacency matrices are arrays of CPMs. For $0 \leq \tau<t$, if we delete $\tau$ column-blocks from $\mathbf{H}_{c}=\mathbf{B}_{c}(\mathbf{P})$, we obtain a $t \times(t-\tau)$ array $\mathbf{H}_{c}(t, t-\tau)$ of CPMs of size $t \times t$ which is a $t^{2} \times t(t-\tau)$ matrix. $\mathbf{H}_{c}(t, t-\tau)$ is the line-point adjacency matrix of a partial geometry $\operatorname{PaG}_{c}(t, t-\tau, t-\tau-1)$ which is a subgeometry of the $\mathrm{PaG}_{c}(t, t, t-1)$.

Notice that all the entries in any row or column, other than the 0-th row and column, are different, since the order of $\beta$ is a prime. By removing the 0 -th row and column from $\mathbf{B}_{c}$, we obtain a Latin square of order $t-1$.

\section{B. $Q C$-LDPC Codes on the $Q C-P a G_{c}(t, t, t-1)$}

For $1 \leq k, r \leq t$, let $\mathbf{B}_{c}(k, r)$ be a $k \times r$ submatrix of the matrix $\mathbf{B}_{c}$ of (9). CPM-dispersing the entries of $\mathbf{B}_{c}(k, r)$, we obtain a $k \times r$ array $\mathbf{H}_{c}(k, r)$ of CPMs of size $t \times t$ which is a subarray of $\mathbf{H}_{c}$. The array $\mathbf{H}_{c}(k, r)$ is a $k t \times r t$ matrix with column and row weights, $k$ and $r$, respectively. The null space of $\mathbf{H}_{c}(k, r)$ gives a $(k, r)$-biregular QC-LDPC code, denoted by $\mathcal{C}_{c, q c}(k, r)$, of length $r t$ with rate at least $(r-k) / r$. The Tanner graph of $\mathcal{C}_{c, q c}(k, r)$ has girth of at least 6 .

In the following, we give an example to illustrate the construction of a QC-LDPC code with QC-structure based on the $\mathrm{QC}-\mathrm{PaG}_{c}(t, t, t-1)$. 
Example 5. Consider the field $G F\left(2^{7}\right)$. Since $2^{7}-1=127$ is a prime, it cannot be factored. So, we choose $t=127$. Let $\alpha$ be a primitive element of $G F\left(2^{7}\right)$. Then, the set $\mathbf{S}=\left\{1, \alpha, \alpha^{2}, \ldots, \alpha^{126}\right\}$ forms the only cyclic subgroup of GF(127). The order of $\mathbf{S} i$ is 127 . With the choice of $t=127$, we construct a $127 \times 127$ base matrix $\mathbf{B}_{c}=\left[\alpha^{i j}\right]_{0 \leq i, j<127}$ in the form of (9). All the 127 nonzero elements in $G F\left(2^{8}\right)$ appear in each row and each column of $\mathbf{B}_{c}$. Dispersing each entry in $\mathbf{B}_{c}$ into a $127 \times 127$ CPM, we obtain a $127 \times 127$ array $\mathbf{H}_{c}=\mathbf{B}_{c}(\mathbf{P})$ of CPMs of size $127 \times 127$. The array $\mathbf{H}_{c}$ is a $16129 \times 16129$ matrix with both column and row weights 127. This matrix is the line-point adjacency matrix of a $Q C-P a G_{c}(127,127,126)$ with 16,129 points and 16,129 lines with connection number 126.

For $1 \leq k, r \leq t$, let $\mathbf{B}_{c}(k, r)$ be a $k \times r$ submatrix of the base matrix $\mathbf{B}_{c}$. Then, the null space of the CPM-dispersion of $\mathbf{B}_{c}(k, r)$ gives a QC-LDPC code of length $127 r$ whose Tanner graph has girth of at least 6.

Label the rows of the base matrix $\mathbf{B}_{c}$ from 0 to 126 . Suppose we take row-1 to row-6 from $\mathbf{B}_{c}$ to form the following $6 \times 127$ submatrix $\mathbf{B}_{c}(6,127)$ of $\mathbf{B}_{c}$ :

$$
\mathbf{B}_{c}(6,127)=\left[\begin{array}{ccccc}
1 & \alpha & \alpha^{2} & \cdots & \alpha^{126} \\
1 & \alpha^{2} & \left(\alpha^{2}\right)^{2} & \cdots & \left(\alpha^{2}\right)^{126} \\
1 & \alpha^{3} & \left(\alpha^{3}\right)^{2} & \cdots & \left(\alpha^{3}\right)^{126} \\
1 & \alpha^{4} & \left(\alpha^{4}\right)^{2} & \cdots & \left(\alpha^{4}\right)^{126} \\
1 & \alpha^{5} & \left(\alpha^{5}\right)^{2} & \cdots & \left(\alpha^{5}\right)^{126} \\
1 & \alpha^{6} & \left(\alpha^{6}\right)^{2} & \cdots & \left(\alpha^{6}\right)^{126}
\end{array}\right]
$$

Notice that matrix $\mathbf{B}_{c}(6,127)$ given by (10) is actually the parity-check matrix of the $(127,121,7)$ Reed-Solomon code of symbol length 127 over $G F\left(2^{7}\right)$ with minimum distance 7. Dispersing each entry of $\mathbf{B}_{c}(6,127)$ into a $127 \times 127$ CPM, we obtain a $6 \times 127$ array $\mathbf{H}_{c}(6,127)$ of CPMs of size $127 \times 127$ which is a subarray of $\mathbf{H}_{c}$. It is a $762 \times 16129$ matrix with column and row weights 6 and 127, respectively. The rank of this matrix is 757. Then, the null space of $\mathbf{H}_{c}(6,127)$ gives $a(6,127)$-biregular $(16129,15372) Q C$-LDPC code $\mathcal{C}_{c, q c}(6,127)$ with rate 0.953.

The bit and block error performances of this code decoded with the MSA are shown in Fig. 6 (computed with an FPGA decoder). We see that the code achieves a BER of $10^{-15}$ and a block error rate (BLER) of almost $10^{-12}$ without a visible error-floor. It has a beautiful waterfall performance. Fig. [6 shows the error performances of the code decoded with 5, 10 and 50 iterations of the MSA. We see that the decoding of the code converges very fast. At the BER 
of $10^{-12}$, the performance gap between 5 and 50 iterations of the MSA is about $0.5 \mathrm{~dB}$. The performance curves of the code decoded with 10 and 50 iterations of the MSA almost overlap all the way down to the BER of $10^{-12}$.

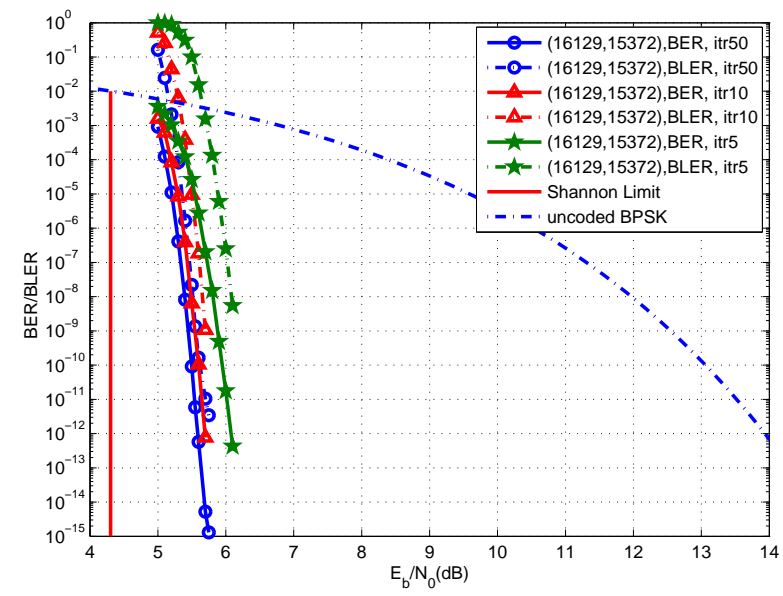

Fig. 6. Performance curves for the $(16129,15372)$ QC-LDPC code given in Example 5

\section{TRAPPING Sets AND Finite GePMEtRies}

Let $\mathcal{G}$ be the Tanner graph of a binary LDPC code where the code is defined as the null space of an $m \times n$ binary matrix. It is assumed that the $n$ vertices on the left of $\mathcal{G}$ are VNs of the code and the $m$ vertices on the right are CNs. The code is to be decoded via a BP decoder, such as the sum-product algorithm (SPA) or the MSA. The behavior of these algorithms depends on the cycle structure of the code as the iterations of the algorithm attempt to converge to a codeword.

A trapping set for the code is defined as follows [25], [37]:

Definition 1. 1) $A(\kappa, \tau)$ trapping set for the code defined by the Tanner graph $\mathcal{G}$ is a subset $\Delta,|\Delta|=\kappa$, of VNs such that the subgraph of $\mathcal{G}$ induced by the set $\Delta$, denoted by $\mathcal{G}[\Delta]$, has exactly $\tau$ odd degree CNs (and an arbitrary number of even degree CNs).

2) The trapping set is said to be elementary if all the associated $C N s$ in $\mathcal{G}[\Delta]$ have degree 1 or 2.

3) The trapping set is called small if $\kappa \leq \sqrt{n}$ ( $n$ is the code length) and $\tau / \kappa \leq 4$. 
A good description of the motivation for these definitions is given in [25], [37] and the reader is referred there for the discussion. In essence, if there is a small trapping set, there is a relatively higher probability that the BP algorithm will fail to converge as it iterates. Beyond trapping sets, the notion of an absorbing set was introduced in [15] to aid in the convergence analysis but as noted, this notion will not be considered in this work.

Trapping sets have been discussed for a variety of LDPC codes obtained from combinatorial structures (in a variety of ways) (see the works [11], [12], [23], [25]-[27], [29]). The question of interest is to discuss them in light of the structure of the finite geometric structure of the code to determine if sharper bounds can be found over those for other codes.

A useful general result is given in Theorem 2 of [11], [12]. It shows that if $\mathcal{G}$ is the Tanner graph of an LDPC code with girth of at least 6 , with VNs of degree $\gamma$, and $\mathcal{G}$ contains a $(\kappa, \tau)$ trapping set and $\kappa<\gamma$, then

$$
\tau \geq(\gamma+1-\kappa) \kappa
$$

A partial geometry code is the dual space to the row space of the $m \times n$ adjacency matrix of the partial geometry $\operatorname{PaG}(\gamma, \rho, \delta)$.

The trapping sets of such codes were examined in [11], [12] and a brief discussion of those results was given. It is noted that the geometric structure of the partial geometry allows a better analysis of the trapping sets over matrices derived from less structured objects. Let $\Delta$ be a subset of VNs of size $\kappa$ and $\mathcal{G}[\Delta]$ the subgraph of the Tanner graph of a code generated by a partial geometry. The properties of the trapping set depends on the structure of this subgraph induced by $\Delta$. Since the CNs generated by $\Delta$ correspond to lines through the points of $\Delta$, it will generate a $(\kappa, \tau)$ trapping set if there are precisely $\tau$ lines in $\mathcal{G}[\Delta]$ passing through an odd number of points of $\Delta$. Let $m_{i}$ be the number of lines of $\mathcal{G}[\Delta]$ passing through $i$ points of $\Delta$ (which is the number of CNs of degree $i$ ). Then:

Theorem 3. ( [12. Theorem 3]) Let $\mathcal{G}_{P a G}$ be the Tanner graph of a $\mathrm{PaG}(\gamma, \rho, \delta)$. If $\Delta$ is a $(\kappa, \tau)$ trapping set and $\kappa \leq \gamma$ then:

$$
\tau \geq(\gamma+1-\kappa) \kappa+\sum_{i \text { odd }}(i-1)^{2} m_{i}+\sum_{i \text { even }} i(i-2) m_{i}
$$

and equality holds if $\delta=\rho$ and the sums go to $2\lfloor(\kappa+1) / 2\rfloor-1$ and $2\lfloor\kappa / 2\rfloor$, respectively.

Note that the first term on the right hand side of this expression is the general bound noted 
earlier. Also the number of edges in the graph generated by a given set of $\kappa$ VNs is, by definition of the partial geometry, $\kappa \gamma$. Hence

$$
\sum_{\text {all } i} i m_{i}=\kappa \gamma \text { and } \sum_{\text {odd } i} m_{i}=\tau
$$

In the particular case of a net the bound can be improved somewhat. Let $L_{0}, L_{1}, \ldots, L_{\rho-1}$ be a set of parallel lines in $\operatorname{PaG}(\gamma, \rho, \gamma-1)$ and for a set $\Delta$ of $\mathrm{VNs}$ let $\kappa_{i}=\left|\Delta \cap L_{i}\right|$. Then:

Theorem 4. If the set $\Delta$ of VNs of a net is a $(\kappa, \tau)$ trapping set, then

$$
\begin{aligned}
\tau & \geq(\gamma-1) \kappa-\kappa^{2} \\
& +\sum_{i=1}^{\rho} \kappa_{i}^{2}+\mid\left\{\ell: 1 \leq \ell \leq \rho, \kappa_{\ell} \text { odd }\right\} \mid
\end{aligned}
$$

It is noted that the bound agrees with the previous one whenever $\kappa_{i} \leq 2$ for all $i$ and improves on it in other cases. A lower bound on the average size of $\tau$ is also given in [12, Corollary 1].

It was observed [29] that the structure of the partial geometric code allows comments on trapping sets for certain types of sets of VNs and a few of these comments are noted here.

A partial ovoid of a partial geometry $\operatorname{PaG}(\gamma, \rho, \delta)$ is a set $\mathbf{S}$ of points (VNs) such that every line in the geometry is incident with at most one point of S. Such a set can have at most $1+(\gamma-1)(\rho-1)$ points. Thus CNs (lines) corresponding to such a set of size $\kappa$ has $\kappa \gamma$ lines that intersect the $\kappa$ VNs and each such $\mathrm{CN}$ has degree 1. Hence $m_{1}=\kappa \gamma$ and all other $m_{i}^{\prime} s$ are 0 . The above bounds are difficult to work with in this case but in the case that $t>3$ the ratio $\kappa / \tau=1+t>4$ and such a set of VNs cannot be a small trapping set.

Consider a set of $\kappa \mathrm{VNs}$ that are colinear, i.e., one line contains all $\kappa$ points. The subgraph generated by such a configuration has $\tau=(\gamma-1) \kappa+1 \mathrm{CNs}, m_{\kappa}=1, m_{1}=\kappa(\gamma-1)$ and $\tau / \kappa=(\gamma-1)+1 / \kappa$. For $\gamma>4$ this does not correspond to a small trapping set.

From these arguments it is clear that the smallest trapping sets will arise from $\mathrm{CN}$ sets that contain large numbers of lines between the $\tau$ points. An extreme case of this is a clique. A clique of a partial geometry is a set of VNs which are mutually colinear. A clique of size $\kappa$ in the partial geometry will generate a subgraph with $(\gamma-\kappa) \kappa=m_{1}$ CNs of degree 1 and $\left(\begin{array}{l}\kappa \\ 2\end{array}\right)=m_{2}$ of degree 2 . The maximum size of a clique for a given partial geometry is not known, but, depending on the values of $\tau, \gamma$ and $\kappa$, they might form a significant trapping set since, from the above argument, assuming a clique of size $\kappa$ exists, $\tau / \kappa=\rho-\kappa$. A clique of size $>2$ is not possible for GQs since it implies forbidden triangles. 
The cases above are extreme but serve as demonstrations that the bounds on the sizes of the trapping sets might be sharpened for the case of partial geometries and GQs.

\section{EXPANSION PROPERTIES OF THE GRAPHS OF PARTIAL GEOMETRIES}

The notion of expansion in coding theory originated in the work of Tanner [45] (under the name of the strongly related concept of superconcentrators). That work also discovered the important relationship between the second largest eigenvalue of the adjacency graph of the code and its expansion properties, a relationship that has been widely exploited by mathematicians and computer science researchers since that time. These issues are examined further here.

Codes that can be encoded and decoded in linear time can be constructed by means of codes with suitable expansion properties [39], the expander codes, and such codes and their graphs have received considerable attention over the past two decades. The codes were not LDPC codes and their decoding algorithm was not a message-passing one. However, it can be argued [45] that expansion properties are also of interest in belief propagation decoding algorithms for LDPC codes since the notion of graph expansion can be interpreted as a measure of connectivity and randomness of the graph, desirable properties for efficient decoding properties. Tanner used such arguments in his seminal paper [44]. In [46] he showed that the minimum distance properties of graph-based codes with small second eignevalue relative to the largest eigenvalue were also good. However, it is in the work of Burshtein and Miller [7] that a more direct relationship between expander graphs and the performance of message-passing decoding algorithms is established. That work uses expander-based arguments to establish that for sufficiently long block lengths, once a message-passing algorithm corrects a sufficiently large fraction of errors, it will eventually correct all errors. The argument considers Gallager hard and soft decoding algorithms but will be applicable for a wider class as well.

Since the second eigenvalue property is important for codes from several points of view, it seems worthwhile to examine the expansion properties of the graphs from partial geometries, a problem that is addressed in this section, with the thought of using this property as a possible distinguisher of codes for further examination. The extent to which these arguments are viable would have to be confirmed with simulation.

The eigenvalues of graphs associated with partial geometries are well known and the contribution of this section is to modify the known results to the purpose of interest, namely the 
expansion properties of graphs from these geometries. A brief overview of the key ideas involved is given.

The majority of works on this topic consider regular graphs, graphs with each vertex having the same degree. Our interest is solely in the biregular bipartite case, defined as bipartite graphs with $n$ left vertices, each of constant degree $c$ and $m$ right vertices, each of degree $d$. When $m$ and $n$ are understood, we refer to this as a $(c, d)$-biregular bipartite graph. The recent work of [18] is an important contribution to this problem as it is the first work known to the authors that considers the expansion properties of these graphs, apart from the original seminal work of Tanner [45]. Large classes of such graphs have been considered in the literature as Tanner graphs associated with partial geometries and other combinatorial configurations. Much of the work on LDPC codes is in fact on such biregular graphs.

The notion of graph expansion is defined in several related ways. The following definition [18] will be used since other results of that work will be of interest. The same notion was used in the original work of Tanner [45], which also considered biregular bipartite graphs. Let a general graph $\mathcal{G}=(\mathbf{V}, \mathbf{E})$ (not necessarily biregular bipartite) with set of vertices $\mathbf{V}$ and edges $\mathbf{E}$ be a connected graph with no self loops. Define the boundary $\delta \mathbf{X}$ of a subset $\mathbf{X} \subset \mathbf{V}$ with $|\mathbf{X}| \leq \alpha|\mathbf{V}|$ as the set of neighbors of $\mathbf{X}$. Define the expansion coefficient $c(\alpha)$ for $|\mathbf{X}| \leq \alpha|\mathbf{V}|$, for some positive fraction $\alpha$, of the graph by:

$$
c(\alpha)=\min _{\phi \neq \mathbf{X} \subset \mathbf{V}} \frac{|\delta \mathbf{X}|}{\min \{|\mathbf{X}|,|\mathbf{V} \backslash \mathbf{X}|\}},
$$

for set $\mathbf{X} \subset \mathbf{V}$. Interest is often in the case where $\alpha<1 / 2$ where the minimization in the denominator is not necessary.

In the case that $\mathcal{G}=(\mathbf{V}, \mathbf{E})$ is a $k$-regular (each vertex is of degree $k$ ), $|\mathbf{V}|=n$ ), and let $\mathbf{A}=\left[a_{i j}\right]_{0 \leq i, j<n}$ be its $n \times n$ adjacency matrix (i.e., its point-point adjacency matrix where $a_{i, j}=1$ if vertex $v_{i}$ is connected to vertex $v_{j}$ ). The matrix $\mathbf{A}$ will have $n$ real eigenvalues which are listed in decreasing order

$$
\mu_{0} \geq \mu_{1} \geq \cdots \geq \mu_{n-1}
$$

and $n$ orthogonal eigenvectors.

If $\mathcal{G}$ is $k$-regular then $\mu_{0}=k$ and $\mu_{n-1}=-\mu_{0}$ if and only if it is bipartite. It can be shown that for any family of $k$-regular connected graphs with number of vertices tending to infinity 
will have

$$
\liminf _{n \rightarrow \infty} \mu_{1} \geq 2 \sqrt{k-1}
$$

A finite connected $k$-regular graph will be called Ramanujan if all of its eigenvalues other than $\pm k$ satisfy the bound

$$
\mu \leq 2 \sqrt{k-1}
$$

Such a graph is described as having a "small" second eigenvalue. The amount of literature on the search for graphs with this property is very large.

As noted, the interest of much of the theory on LDPC codes is focussed on the construction of $(c, d)$-biregular bipartite graphs for use as Tanner graphs of the code and the recent work [18] is precisely on the expansion properties of such graphs. This section considers that work in the light of the graphs from partial geometries, all of whose eigenvalues are known.

Let $\mathbf{V}_{1}$ be the set of $c$-regular $\mathrm{VNs}$ and $\mathbf{V}_{2}$ the set of $d$ regular CNs. Interest is in the case where the expansion in the previous definitions is for sets of VNs and $\mathrm{X} \subset \mathrm{V}_{1}$ and its boundary is a subset of the CNs $\mathrm{V}_{2}$ and bounds on the resulting expansion coefficients is in terms of the eigenvalues of the adjacency matrix. This case was included in the interesting recent work [18] and several results from that work will be of interest here. That work defined the $(c, d)$-biregular bipartite graph $\mathcal{G}$ to be Ramanujan if

$$
\mu_{1}(\mathcal{G}) \leq \sqrt{c-1}+\sqrt{d-1}
$$

a natural extension of the regular graph case by setting $c=d$, although not theoretically justified.

For coding applications, the parity check matrix $\mathbf{H}$ is often the $m \times n$ matrix derived from a combinatorial configuration or finite geometry, where the $m$ rows of $\mathbf{H}$ are identified with the blocks of the configuration or lines of the geometry and the columns of $\mathbf{H}$ with the points. It can be described as the line-point adjacency matrix of the structure. The work of [18] refers to this matrix as the transfer matrix.

In terms of this matrix $\mathbf{H}$, the adjacency matrix of the configuration or geometry which is $n \times n$, a point-point adjacency matrix, (and not using the blocks as graph vertices) is easily seen to be

$$
\mathbf{A}_{1}=\mathbf{H}^{T} \mathbf{H}-\gamma \mathbf{I}
$$

where there are $\gamma$ lines intersecting a point and $\mathbf{I}$ is a $n \times n$ identity matrix. 
The related adjacency matrix $\mathbf{A}$ which includes both the left vertices of the Tanner graph (VNs of the code) and the right vertices (CNs of the code), is of the form

$$
\mathbf{A}=\left[\begin{array}{cc}
\mathbf{O} & \mathbf{H}^{T} \\
\mathbf{H} & \mathrm{O}
\end{array}\right]
$$

This is the matrix and graph whose expansion properties are of interest. Of course in the case of a $(c, d)$-biregular bipartite graph, the rows of the $m \times n$ matrix $\mathbf{H}$ have weight $d$ and columns have weight $c$. Note that

$$
\mathbf{A}^{2}=\left[\begin{array}{cc}
\mathbf{H}^{T} \mathbf{H} & \mathbf{O} \\
\mathbf{O} & \mathbf{H H}^{T}
\end{array}\right]
$$

As discussed in [8], [21], the eigenvalues of the adjacency matrix of graphs of partial geometries are well known. Such combinatorial structures correspond to two class association schemes and strongly regular graphs and the eigenvalues of the adjacency matrix of the graphs of the $\operatorname{PaG}(\gamma, \rho, \delta) \mathbf{A}_{1}$ of (11) are given by:

$$
\begin{aligned}
(\rho-1) \gamma & \text { multiplicity } 1 \\
\rho-1-\delta & \text { multiplicity } \frac{\gamma \rho(\gamma-1)(\rho-1)}{\delta(\gamma+\rho-1-\delta)} \\
-\gamma & \text { multiplicity } \frac{(\rho-1)(\rho-\delta)((\gamma-1)(\rho-1)+\delta)}{\delta(\gamma+\rho-1-\delta)}
\end{aligned}
$$

It is clear [21] that the eigenvalues of $\mathbf{H}^{T} \mathbf{H}$ are those of the matrix $\mathbf{A}_{1}$ of (11), with $\gamma$ added to each eigenvalue, and with the same multiplicities, i.e., the eigenvalues of $\mathbf{H}^{T} \mathbf{H}$ are $\gamma \rho$, $\gamma+\rho-1-\delta$ and 0 .

It is noted in [45] that if $\mathbf{x}$ is an eigenvector of $\mathbf{H}^{T} \mathbf{H}$ with eigenvalue $\lambda \neq 0$ then $\mathbf{x}^{\prime}=\mathbf{H x}$ is an eigenvector of $\mathbf{H H}^{T}$ with the same eigenvalue. Thus $\mathbf{H}^{T} \mathbf{H}$ and $\mathbf{H} \mathbf{H}^{T}$ have the same eigenvalues (with different multiplicities). It is not difficult to show that if the eigenvalues of the matrix of interest $\mathbf{A}$ in (12) are $\left\{\mu_{i}, i=0,1, \ldots, n-1\right\}$, then the eigenvalues of $\mathbf{H}^{T} \mathbf{H}$ are $\left\{\mu_{i}^{2}, i=0,1, \ldots, n-1\right\}$ and the expansion properties of the graph are determined by the "second" eigenvalue $\mu_{1}$.

As $\mathbf{A}$ is a real symmetric matrix we have the rank of $\mathbf{A}, r_{\mathbf{A}}$, is equal to the rank of $\mathbf{A}^{2}$ and from (12), $r_{\mathbf{A}}=2 r_{\mathbf{H}}$. The eigenvalues of $\mathbf{A}^{2}$ are those of $\mathbf{H}^{T} \mathbf{H}$ which are the same (in magnitude, not necessarily in multiplicity) as those of $\mathbf{H H}^{T}$. Thus, arguing in the reverse from the previous paragraph, the eigenvalues of the matrix $\mathbf{A}$ of (12) are $\pm \sqrt{\gamma \rho}, \pm \sqrt{\gamma+\rho-1-\delta}$ and 0 . Thus the second eigenvalue is $\sqrt{\gamma+\rho-1-\delta}$. 
The expansion coefficient of any $(c, d)$-biregular bipartite graph is given by [18], [45]

$$
c(\alpha) \geq \frac{c^{2}}{\alpha c d+\mu_{1}^{2}(1-\alpha)}
$$

and the importance of the magnitude of the second eigenvalue is seen, i.e., the smaller the size of $\mu_{1}$ the larger the expansion of the graph.

Such information is used in [46] and [21] to determine bounds on the minimum distance of the codes with parity-check matrix $\mathbf{H}$. The interest here is in the expansion properties of the code with parity-check matrix $\mathbf{H}$. Since the second eigenvalue of the matrix $\mathbf{A}$ is $\sqrt{\gamma+\rho-1-\delta}$, this suggests that the graphs of partial geometries with $\delta$ as large as possible, while still being partial geometries, will have the best expansion properties, i.e., $\delta=\gamma-1$ corresponding to nets. Notice that the two classes of partial geometries constructed in this paper are two classes of nets. For the first class, $\gamma=\rho=p$ and $\delta=p-1$, and for the second class, $\gamma=\rho=t$ and $\delta=t-1$. Hence, they have good expansion properties. While the parameters of the partial geometry, $\gamma, \rho$ and $\delta$, are not independent, the conclusion is interesting and worthy of further consideration.

For interest, to conclude this section, the ratio of $\mu_{1}^{2} / \mu_{\max }^{2}$ is compared for biregular bipartite graphs and $k$-regular graphs. From the discussion, for $k$-regular graphs, for the Ramanujan case,

$$
\mu_{1}^{2} / \mu_{\max }^{2}=2(k-1) / k^{2} \approx 2 / k
$$

while for $(\gamma, \rho)$-biregular bipartite graphs from partial geometries the ratio is

$$
\mu_{1}^{2} / \mu_{\max }^{2}=\frac{\gamma+\rho-(\delta+1)}{\gamma \rho}=\frac{1}{\rho}+\frac{1}{\gamma}-\frac{(\delta+1)}{\gamma \rho} .
$$

If parameters can be chosen so that $\rho \approx \gamma \approx k$ and $\delta$ small, the two ratios are similar. For larger values of $\delta$, however, such as for nets, the ratio of the eigenvalues of the partial geometry is approximately $1 / \rho$, suggesting graphs with large $\rho$ and $\delta$ would have better expansion.

\section{CONCLUSION AND REMARKS}

Several aspects of codes derived from partial geometries have been considered. New results on codes from partial geometries were given, including an interesting characterization of them in terms of arrays of cyclic permutation matrices and two new and simple constructions. The trapping sets of codes from partial geometries were also investigated using the geometric properties of their constructions. Finally, comments were given on the expansion properties of graphs from partial geometries. 


\section{REFERENCES}

[1] S. Abu-Surra, D. Divsalar, and W. E. Ryan, "Enumerators for protograph-based ensembles of LDPC and generalized LDPC codes," IEEE Trans. Inform. Theory, vol. 57, no. 2, pp. 858-886, Feb. 2011.

[2] B. Bagchi and N. S. N. Sastry, "Even order inversive planes, generalized quadrangles and codes," Geomtriae Dedicata, vol. 22, no. 2, pp. 137-147, Feb. 1987.

[3] B. Bagchi and N. S. N. Sastry, "Codes associated with generalized polygons," Geometriae Dedicata, vol. 27, no. 1, pp. 1-8, Jul. 1988.

[4] B. Bagchi, A. E. Brouwer, and A. Willbrink, "Notes on binary codes related to the $O(5, q)$ generalized quadrangle for odd q," Geom. Dedicata, vol. 39, no. 3, pp. 339-355, Sep. 1991.

[5] L. M. Batten, Combinatorics of Finite Geometries, 2nd ed. Cambridge, UK: Cambridge Univ. Press, 1997.

[6] R. C. Bose, "Strongly regular graphs, partial geometries and partially balanced designs," Pacif. J. Math., vol. 13, no. 2, pp. 389-419, Apr. 1963.

[7] D. Burshtein and G. Miller, "Expander graph arguments for message-passing algorithms," IEEE Trans. Inform. Theory, vol. 47, no. 2, pp. 782-790, Feb. 2001.

[8] P. J. Cameron and J. H. van Lint, Designs, Graphs, Codes, and Their Links, Cambridge, UK: Cambridge Univ. Press, 1991.

[9] R. D. Carmichael, Introduction to the Theory of Groups of Finite Orders, New York, NY: Dover, 1956.

[10] J. Chen and M. P. C. Fossorier, "Near optimum universal belief propagation based decoding of low-density parity-check codes," IEEE Trans. Inform. Theory, vol. 50, no. 3, pp. 406-414, Mar. 2002.

[11] Q. Diao, Y. Y. Tai, S. Lin, and K. Abdel-Ghaffar, "LDPC codes on partial geometries: Construction, trapping sets structure, and puncturing," IEEE Trans. Inform. Theory, vol. 59, no. 12, pp. 7898-7914, Sep. 2013.

[12] Q. Diao, Y. Y. Tai, S. Lin, and K. Abdel-Ghaffar, "Trapping set structure of finite geometry LDPC codes," in Proc. IEEE Int. Symp. Inf. Theory, Cambridge, MA, Jul. 1-6, 2012, pp. 3088-3092.

[13] D. Divsalar, S. Dolinar, and C. R. Jones, "Construction of protograph LDPC codes with linear minimum Distance," in Proc. IEEE Int. Symp. Inf. Theory, Seattle, WA, Jul. 9-14, 2006, pp. 664-668.

[14] D. Divsalar, S. Dolinar, C. R. Jones, and K. Andrews, "Capacity-approaching protograph codes," in IEEE J. Sel. Areas Commun., vol. 27, no. 6, pp. 876-888, Aug. 2009.

[15] L. Dolocek, Z. Zhang, V. Anantharam, M. Wainwright, and B. Nikolić, "Analysis of absorbing sets and fully absorbing sets of array-based LDPC codes," IEEE Trans. Inform. Theory, vol. 56, pp. 181-201, Jan. 2010.

[16] R. G. Gallager, "Low density parity-check codes," IRE Trans. Inform. Theory, vol. IT-8, no. 1, pp. 21-28, Jan. 1962.

[17] C. Godsil and G. Royle, Algebraic Graph Theory, Springer-Verlag, GTM 207, 2001.

[18] T. Høholdt and H. Janwaa, "Eigenvalues and expansion of bipartite graphs," Designs, Codes and Cryptography, vol. 65, no. 3, pp. 259-273, Dec. 2012.

[19] X. -Y Hu, E. Eleftheriou, and D. -M Arnold, "Progressive edge-growth Tanner graphs," in Proc. IEEE Glob. Commun. Conf., San Antonio, TX, Nov. 25-29, 2001, pp. 995-1001.

[20] J. R. H. Hutton, LDPC Codes from Semipartial Geometries, Ph.D. Dissertation: University of Sussex, UK, 2011.

[21] S. J. Johnson and S. R. Weller, “Codes for iterative decoding from partial geometries," IEEE Trans. Commun., vol. 52, no. 2, pp. 236-243, Feb. 2004.

[22] N. Kamiya, "High-rate quasi-cyclic low-density parity-check codes derived from finite affine planes," IEEE Trans. Inform. Theory, vol. 53, no. 4, pp. 1444-1459, Apr. 2007.

[23] J. -L Kim, K. E. Mellinger, and L. Storme, "Small weight codewords in LDPC codes defined by (dual) classical quadrangles," Designs, Codes and Cryptography, vol. 42, no. 1, pp. 73-92, Jan. 2007. 
[24] Y. Kou, S. Lin, and M. P. C. Fossorier, "Low-density parity-check codes based on finite geometries: A rediscovery and new results," IEEE Trans. Inform. Theory, vol. 47, no. 7, pp. 2711-2736, Nov. 2001.

[25] S. Laender and O. Milenkovic, "LDPC codes based on Latin squares: Cycle structure, stopping set, and trapping set analysis,” IEEE Trans. Commun., vol. 55, no. 2, pp. 303-312, Feb. 2007.

[26] S. Laedner and O. Milenkovic, "Algorithmic and combinatorial analysis of trapping sets in structured LDPC codes," Int'l Conf. Wireless, Networks, Communications and Mobile Computing, Maui, HI, Jun. 13-16, 2005, pp. 630-635.

[27] S. Laedner, O. Milenkovic, and J.B. Huber, "Characterizations of small trapping sets in LDPC codes from Steiner triple systems," in Proc. Turbo Codes and Iterative Information Processing, Brest, France, Sep. 6-10, 2010, pp. 93-97.

[28] X. Li, C. Zhang, and J. Shen, "Regular LDPC codes from semipartial geometries," Acta Appl. Math., vol. 102, no. 1, pp. 25-35, May 2008.

[29] S. Lin, Q. Diao, and I.F. Blake, "Error floors and finite geometries," in Proc. Turbo Codes and Iterative Information Processing, Bremen, Germany, Aug. 18-22, 2014, pp. 42-46.

[30] Z. Liu and D. A. Pados, "Generalized quad, hex and octagon LDPC codes," in Proc. IEEE Glob. Commun. Conf., St. Louis, Missouri, Nov. 28-Dec. 2, 2005, pp. 1137-1141.

[31] Z. Liu and D. A. Pados, "LDPC codes from generalized polygons," IEEE Trans. Inform. Theory, vol. 51, no. 2, pp. 3890-3898, Nov. 2005.

[32] D. MacKay and R. Neal, "Near Shannon limit performance of low density parity-check codes," Electro. Lett., vol. 32, no. 18, pp. 1645-1646, Aug. 1996.

[33] D. MacKay, "Good error-correcting codes based on very sparse matrices," IEEE Trans. Inform. Theory, vol. 45, no. 2, pp. 399-431, Mar. 1999.

[34] H. Mann, Analysis and Design of Experiments, New York, NY: Dover, 1949.

[35] S. Payne and J. Thas, Generalized Quadrangles, European Mathematical Society, 2nd Edition, 2009.

[36] V. Pepe, L. Storme, and G. Van de Voorde, "On codewords in the dual of classical generalized quadrangles and classical polar spaces," Disc. math., vol. 310, no. 22, pp. 3132-3148, Nov. 2010.

[37] T. Richardson, "Error floors and LDPC codes," in Proc. 41st Allerton Conf. Communication, Control, and Computing, Monticello, IL, Oct. 1-3, 2003, pp. 1426-1435.

[38] N. S. N. Sastry and P. Sin, "The code of a regular generalized quadrangle of even order," in Proc. Symp. Pure Math., vol. 63, pp. 485-496, Jan. 1998.

[39] M. Sipser and D. Spielman, "Expander codes," IEEE Trans. Inform. Theory, vol. 42, no. 6, pp. 1710-1722, Nov. 1996.

[40] P. Sin and Q. Xiang, "On the dimensions of certain LDPC codes based on q-regular bipartite graphs," IEEE Trans. Inform. Theory, vol. 52, no. 8, pp. 3735-3737, Aug. 2006.

[41] A. P. Street and D. J. Street, Combinatorics of Experimental Designs, New York, NY: Oxford, Clarendon Press, 1987.

[42] H. Tang, J. Xu, Y. Kou, S. Lin, and K. Abdel-Ghaffar, "On algebraic construction of Gallager and circulant low density parity check codes," IEEE Trans. Inform. Theory, vol 50, no. 6, pp. 1269-1279, Jun. 2004.

[43] H. Tang, J. Xu, S. Lin, and K. Abdel-Ghaffar, "Codes on finite geometries," IEEE Trans. Inform. Theory, vol. 51, no. 2, pp. 572-596, Feb. 2005.

[44] R. M. Tanner, "A recursive approach to low complexity codes," IEEE Trans. Inform Theory, vol. IT-27, no. 5, pp. 533-547, Sep. 1981.

[45] R. M. Tanner, "Explicit concentrators from generalized N-gons," SIAM J. Alg. Dics. Meth., vol. 5. no. 3, pp. 287-293, 1984.

[46] R. M. Tanner, "Minimum distance bounds by graph analysis," IEEE Trans. Inform. Theory, vol. 47, no. 2, pp. 808-821, Feb. 2001. 
[47] J. Thorpe, "Low-density parity-check codes constructed from protographs," IPN Progress Report, pp. 42-154, Aug. 2003.

[48] P. Vandendriessche, "Some low-density parity-check codes derived from finite geometries," Designs, Codes and Cryptography, vol. 54, pp. 287-297, Sep. 2009.

[49] P. Vandendriessche, "LDPC codes arising from partial and semipartial geometries," in Int. Workshop Coding and Cryptography, Paris, France, Apr. 11-15, 2011, pp. 419-428.

[50] H. van Maldeghem, Generalized Polygons, Birkhäuser Verlag, 1998.

[51] P. Vontobel, Algebraic Coding for Iterative Decoding, Ph.D. Dissertation, Eidgenössische Technische Hochschule (ETH), Zürich, 2003.

[52] P. Vontobel and R. M. Tanner, "Construction of codes based on finite generalized quadrangles for iterative decoding," in Proc. IEEE Int. Symp. Inf. Theory, Washington, DC, Jun. 24-29, 2001, p. 223.

[53] J. Xu, L. Chen, I. Djurdjevic, S. Lin, and K. Abdel-Ghaffar, "Construction of regular and irregular LDPC codes: Geometry decomposition and masking," IEEE Trans. Inform. Theory, vol. 53, no. 1, pp. 121-134, Jan. 2007.

[54] L. Zhang, Q. Huang, S. Lin, and Ian F. Blake, "Quasi-cyclic LDPC codes: An algebraic construction, rank analysis and codes on Latin squares," IEEE Trans. Commun., vol. 58, no. 11, pp. 3126-3139, Sep. 2010.

[55] Q. Diao, Q. Huang, S. Lin, and K. Abdel-Ghaffar, "A transform approach for analyzing and constructing quasi-cyclic low-density parity-check codes," in Proc. Inf. Theory Applic. Workshop, San Diego, CA, Feb. 6-11, 2011, pp. 1-8. 